\title{
Neural Mechanisms Underlying High-Frequency Vestibulocollic Reflexes In Humans And Monkeys
}

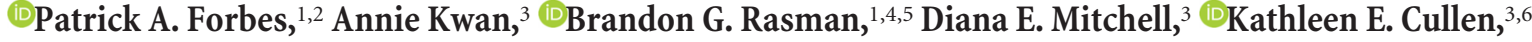 \\ and Jean-Sébastien Blouin ${ }^{5}$ \\ ${ }^{1}$ Department of Neuroscience, Erasmus University Medical Centre, Rotterdam, 3015 GD, The Netherlands, ${ }^{2}$ Department of Biomechanical Engineering, \\ Faculty of Mechanical, Maritime and Materials Engineering, Delft University of Technology, Delft, 2628 CD, The Netherlands, ${ }^{3}$ Department of Biomedical \\ Engineering, McGill University, Montreal, Quebec, H3G 1Y6, Canada, ${ }^{4}$ School of Physical Education, Sport and Exercise Sciences, University of Otago, \\ Dunedin, 9016, New Zealand, ${ }^{5}$ School of Kinesiology, Djavad Mowafaghian Centre for Brain Health, and Institute for Computing, Information and Cognitive \\ Systems, University of British Columbia, British Columbia, V6T 1Z1, Canada, and ${ }^{6}$ Department of Biomedical Engineering, Johns Hopkins University, \\ Baltimore, 21205, Maryland
}

The vestibulocollic reflex is a compensatory response that stabilizes the head in space. During everyday activities, this stabilizing response is evoked by head movements that typically span frequencies from 0 to $30 \mathrm{~Hz}$. Transient head impacts, however, can elicit head movements with frequency content up to $300-400 \mathrm{~Hz}$, raising the question whether vestibular pathways contribute to head stabilization at such high frequencies. Here, we first established that electrical vestibular stimulation modulates human neck motor unit (MU) activity at sinusoidal frequencies up to $300 \mathrm{~Hz}$, but that sensitivity increases with frequency up to a low-pass cutoff of $\sim 70-80 \mathrm{~Hz}$. To examine the neural substrates underlying the low-pass dynamics of vestibulocollic reflexes, we then recorded vestibular afferent responses to the same electrical stimuli in monkeys. Vestibular afferents also responded to electrical stimuli up to $300 \mathrm{~Hz}$, but in contrast to MUs their sensitivity increased with frequency up to the afferent resting firing rate $(\sim 100-150 \mathrm{~Hz})$ and at higher frequencies afferents tended to phase-lock to the vestibular stimulus. This latter nonlinearity, however, was not transmitted to neck motoneurons, which instead showed minimal phase-locking that decreased at frequencies $>75 \mathrm{~Hz}$. Similar to human data, we validated that monkey muscle activity also exhibited low-pass filtered vestibulocollic reflex dynamics. Together, our results show that neck MUs are activated by high-frequency signals encoded by primary vestibular afferents, but undergo low-pass filtering at intermediate stages in the vestibulocollic reflex. These high-frequency contributions to vestibular-evoked neck muscle responses could stabilize the head during unexpected head transients.

Key words: high frequency; human; neck motor units; non-human primates; vestibular afferents; vestibulocollic reflex

\section{Significance Statement}

Vestibular-evoked neck muscle responses rely on accurate encoding and transmission of head movement information to stabilize the head in space. Unexpected transient events, such as head impacts, are likely to push the limits of these neural pathways since their high-frequency features $(0-300 \mathrm{~Hz})$ extend beyond the frequency bandwidth of head movements experienced during everyday activities $(0-30 \mathrm{~Hz})$. Here, we demonstrate that vestibular primary afferents encode high-frequency stimuli through frequency-dependent increases in sensitivity and phase-locking. When transmitted to neck motoneurons, these signals undergo low-pass filtering that limits neck motoneuron phase-locking in response to stimuli $>75 \mathrm{~Hz}$. This study provides insight into the neural dynamics producing vestibulocollic reflexes, which may respond to high-frequency transient events to stabilize the head.

\section{Introduction}

To interact with the world, humans rely on accurate encoding of biologically-relevant events by sensory receptors and afferents, as

Received June 20, 2019; revised Dec. 30, 2019; accepted Jan. 7, 2020.

Author contributions: P.A.F., A.K., K.E.C., and J.-S.B. designed research; P.A.F., A.K., B.G.R., D.E.M., K.E.C., and J.-S.B. performed research;P.A.F. and B.G.R. analyzed data; P.A.F. and B.G.R. wrote the first draft of the paper; P.A.F., B.G.R., D.E.M., K.E.C., and J.-S.B. edited the paper; P.A.F., K.E.C., and J.-S.B. wrote the paper.

This work was funded by the Natural Sciences and Engineering Research Council of Canada (Grant RGPIN: 356026-13 to J.-S.B.), the National Institute on Deafness and Other Communication Disorders at the National Institutes of Health (Grants R01-DC002390 and R01-DC013069 to K.E.C.), and the Canadian Institutes of Health Research (K.E.C.). P.A.F. received funding from the European Union's Seventh Framework Programme for Research, Technological Development and Demonstration under the People Programme (Marie Curie Actions Grant 624158) and the well as faithful transmission of these signals to motoneurons to ensure accurate motor control. The vestibular system plays a crucial role in these processes via its rate and temporal coding (Jamali et al., 2016, 2019), providing information about head

Netherlands Organization for Scientific Research (NW0 Grant 016.Veni.188.049). B.G.R. received a graduate studen scholarship from the Natural Sciences and Engineering Research Council of Canada. We thank Dale Roberts for excellent technical assistance, and Pum Wibiinsaksakul, Omid Zobeiri and Lexi Lowe for their assistance with experiments.

The authors declare no competing financial interests.

Correspondence should be addressed to Patrick A. Forbes at p.forbes@erasmusmc.nl.

https://doi.org/10.1523/JNEUROSCI.1463-19.2020

Copyright $\odot 2020$ the authors 


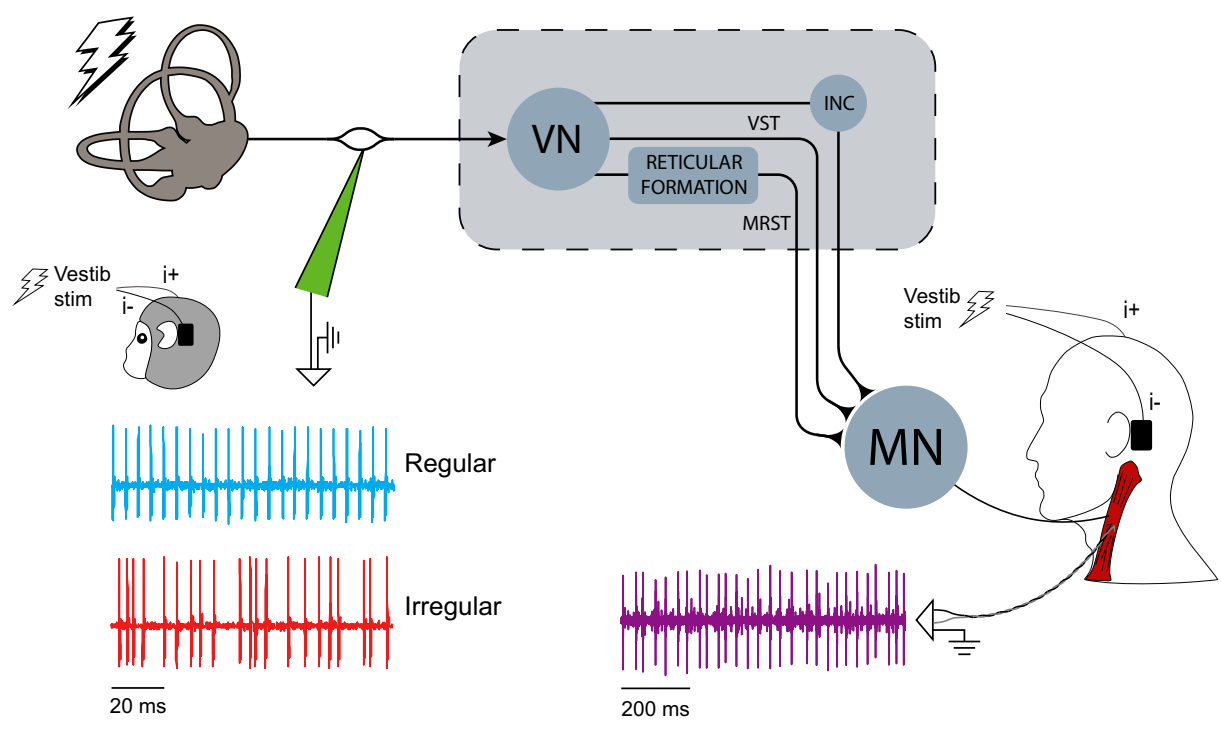

Figure 1. Recording arrangement and neural pathways of the electrically evoked vestibulocollic reflex in monkey and human. Extracellular single-unit activity of regular and irregular canal afferents in macaque monkeys was recorded using tungsten electrodes during sinusoidal and stochastic electrical vestibular stimulation. Single MU activity was recorded in human sternocleidomastoid and splenius capitis muscles and multi-unit activity was recorded in human and monkey sternocleidomastoid muscle using fine-wire electrodes during the same electrical stimuli. INC, Interstitial nucleus of Cajal; MN, motoneurons; MRST, medial reticulospinal tract; VN, vestibular nuclei; VST, vestibulospinal tract.

movement and orientation in space that is used to control gaze, balance and navigation (Cullen, 2019). Notably, vestibulospinal reflexes help stabilize the head and body relative to inertial space by sending motor commands to the axial and appendicular muscles that compensate for current head-in-space motion (Wilson and Schor, 1999; Goldberg and Cullen, 2011). These descending signals are produced during daily activities that typically involve head movements up to $30 \mathrm{~Hz}$ (Carriot et al., 2014). However, unexpected transient events, such as those experienced during a fall or impact, are likely to extend to higher frequencies. Indeed, a recent study has established that direct head impacts evoke head kinematic responses as high as $300-400 \mathrm{~Hz}$ (Wu et al., 2016). Thus, the question arises whether muscle responses of vestibular origin can be evoked at the high frequencies comprising unexpected head transients.

The frequency characteristics of motor responses evoked by vestibular stimuli differ markedly across muscles (Forbes et al., 2015). For example, vestibular-evoked responses elicited by transmastoid electrical stimulation contribute to human lower limb muscle activity up to frequencies of $\sim 25 \mathrm{~Hz}$ (Dakin et al., 2007), but show no signs of abating up to $75 \mathrm{~Hz}$ in human neck muscles (Forbes et al., 2013). Computational modeling based on these results has led to the proposal that pathway-dependent neural low-pass filtering contributes to these muscle-specific responses (Forbes et al., 2013). Although this is supported by heterogeneity in the neural dynamics of both primary afferents and central neurons within the vestibular system (Straka et al., 2005; Jamali et al., 2016, 2019), there are several limitations to this proposal. First, estimates of vestibulocollic reflexes evoked by electrical stimulation rely on nonstandard high-pass filtering $(>75 \mathrm{~Hz})$ and rectification of multi-unit electromyography (EMG) signals (Dakin et al., 2014; Forbes et al., 2014). Rectification, in particular, may induce nonlinear distortion of spectral estimates and timing of stimulus-evoked motor unit (MU) responses (Boonstra and Breakspear, 2012; McClelland et al., 2012; Farina et al., 2013). Such distortion would likely be problematic at higher frequencies $(>60 \mathrm{~Hz})$ where the shape of action potentials is more likely to influence frequency responses (Halliday and Farmer, 2010; Ward et al., 2013). Second, the vestibulocollic re- flex has only been examined at frequencies up to $75 \mathrm{~Hz}$ (Forbes et al., 2013). Accordingly, it is unclear whether neural filtering also limits the frequency range of vestibulocollic reflexes.

The aim of the current study was to understand the dynamic responses (sensitivity/phase/coherence) of neck muscles and vestibular afferents evoked by electrical vestibular stimuli (see Fig. 1) at frequencies up to those reported during head impact $(\sim 300$ $\mathrm{Hz}$; Wu et al., 2016). We found that human neck MUs responded to stimuli up to $300 \mathrm{~Hz}$, with sensitivity peaking at $\sim 70-80 \mathrm{~Hz}$. We then examined the neural substrates underlying these muscle responses by characterizing the vestibular afferent activity produced by the same electrical stimuli in macaque monkeys. Primary vestibular afferents also responded to electrical vestibular stimuli up $\sim 300 \mathrm{~Hz}$ but, unlike neck MUs, sensitivity increased with frequency to a plateau at the afferent resting firing rate $(\sim 100-150 \mathrm{~Hz})$. Further, vestibular afferents tended to synchronize (phase-lock) with the increasing stimulus frequency; however, this increased synchronization was not transmitted to neck MUs and phase-locking instead decreased at frequencies $>75 \mathrm{~Hz}$. Finally, cross-species comparison of human and monkey neck muscle responses showed similar vestibulocollic reflex dynamics. Together our results suggest that the responses of neck motoneurons to vestibular inputs are shaped through filtering by intermediate pathways and that their high-frequency components could contribute to head stabilization during unexpected transient events such as falls or head impacts.

\section{Materials and Methods}

\section{Human subject testing}

Eight healthy male subjects (age 27.4 \pm 3.2 years, mass $79.3 \pm 22.2 \mathrm{~kg}$, height $179.6 \pm 4.4 \mathrm{~cm}$ ) with no self-reported history of neurological disorders participated in this study. The experimental protocol was explained before the experiment and all subjects gave written informed consent. The experiment conformed to the Declaration of Helsinki and was approved by the University of British Columbia's Clinical Research Ethics Board.

\section{Single and multi-MU recording}

Muscle activity was recorded primarily from the left sternocleidomastoid with custom-made insulated fine-wire electrodes (Stablohm 800A; Cal- 
ifornia Fine Wire). Two electrodes of slightly different designs were made and inserted for the purposes of recording both single- and multi-unit activity. We consider single MU recordings as the gold standard measurement of neck motoneuron activity because single MU recordings have higher signal-to-noise ratios and improved temporal resolution relative to multi-unit recordings (Johnston et al., 2010). Therefore, if both single and multi-unit responses are in agreement, this would support the use of multi-unit (i.e., indwelling EMG) recordings to represent the influence of vestibular inputs on the activity of multiple MUs at the frequencies of the electrical vestibular stimuli tested here. Throughout the manuscript we refer to single-unit recordings as single MU and multi-unit recordings as multi-unit EMG. Single-unit electrodes were made from paired wires wound together. The ends of the two wires were cut to expose their cross-section $\left(0.05 \mathrm{~mm}^{2}\right)$ to record distinct MU action potentials. For each subject $(n=8)$, we extracted a single MU. Multi-unit electrodes were made from the same material and in the same fashion with the exception that one of the two wires had $5 \mathrm{~mm}$ of insulation removed to allow for the recording of multi-unit potentials. Both electrodes were inserted under ultrasound guidance (Micromaxx; Sonosite) within close proximity of one another and remained superficial to the readily identifiable cleidomastoid subvolume (Kamibayashi and Richmond, 1998). A ground surface electrode (Blue Sensor M; Ambu $\mathrm{A} / \mathrm{S}$ ) was placed on the anterior surface of the left clavicle. Muscle activity signals were amplified $(\times 200$ single-unit, $\times 500$ multi-unit; NeuroLog, Digitimer), band-pass filtered $(10-5000 \mathrm{~Hz})$ and digitized at $20 \mathrm{kHz}$ together with the vestibular stimuli via a digital acquisition board (PXI6289, National Instruments) using a custom LabVIEW software program (National Instruments).

To verify that high-frequency vestibular-evoked responses could be observed in other neck muscles, we recorded single MUs from the right splenius capitis muscle of two additional human male subjects (age 39/30 years, mass $75 / 75 \mathrm{~kg}$, height $182 / 168 \mathrm{~cm}$ ) following similar methods as described above. For these additional data, the same experimental protocol, vestibular stimuli and single MU data analyses (see below) were used.

\section{Protocol}

Subjects were seated with their eyes closed throughout all experiments and performed isometric neck muscle contractions with the head fixed in a helmet (Pro-tec; Vans). The head was pitched up such that Reid's plane (inferior orbital margins to external acoustic meatus) was positioned $\sim 18^{\circ}$ above horizontal. In this head posture, the net rotation vector induced by electrical vestibular stimuli is orthogonal to gravity (see Schneider et al., 2002; Fitzpatrick and Day, 2004; Chen et al., 2019; roll in head-centered coordinates), and results in an inferred interaural acceleration due to the integration of canal/otolith signals with an internal estimate of gravity (Khosravi-Hashemi et al., 2019). The torso was secured to a stationary backboard with Velcro straps to limit body movement during the isometric neck contractions. The experiment was divided into two blocks: single- and multi-unit recordings. Each block was comprised of 15 trials, with each trial lasting $80 \mathrm{~s}$ during which subjects were exposed to different electrical vestibular stimuli (see "Electrical vestibular stimuli" section). For each block, the single- and multiunit signals were passed through three second order high-pass filters each with a $300 \mathrm{~Hz}$ cutoff (model 440, Brownlee Precision). This filtering approach was used to remove the artifact from the measurements during stimulation only for online processing during the experiment (see below).

During the single MU recordings, a single MU waveform was identified on an oscilloscope by the experimenter at the start of each trial before delivering the stimuli. Subjects were given audio feedback of the single MU firing to maintain the MU at a relatively constant firing rate. During multi-unit recordings, subjects first performed a reference muscle contraction to define the target muscle activity for all subsequent trials (Forbes et al., 2014). Subjects lay flat on the floor and lifted their head for $30 \mathrm{~s}$ to generate sternocleidomastoid muscle activity. The resulting multi-unit EMG signal was high-pass filtered offline (phaseless eighthorder Butterworth digital filter, $-3 \mathrm{~dB}$ at $100 \mathrm{~Hz}$ ) and target muscle activity was then calculated as $40 \%$ of the root-mean-square (RMS) to provide subjects with real-time feedback of muscle activity. The target level of muscle activity was sufficient to elicit the electrically-evoked vestibulocollic reflex, which is only observed in active neck muscles (Watson and Colebatch, 1998; Forbes et al., 2014). Subjects were instructed to generate a neck flexion moment by pushing their forehead into the helmet. The analog filtered multi-unit signal was processed in real-time by calculating the RMS (50 ms window), then low-pass filtering $(1 \mathrm{~Hz})$ the RMS for presentation to the experimenter. The subjects were given verbal feedback throughout the trials to help them maintain a constant level of muscle activity. To confirm that there was no difference in the level of muscle activity across all trials, we evaluated the RMS of the filtered EMG using repeated-measures ANOVAs (10 stimulation conditions).

\section{Nonhuman primate testing}

Two male macaque monkeys (Macaca fascicularis, Monkeys $\mathrm{B}$ and $\mathrm{H}$ ) were prepared for chronic extracellular recording of vestibular afferents using aseptic surgical techniques. The surgical preparation and postsurgery protocol for the monkeys followed the procedures described previously (Monkey B: Dale and Cullen, 2013; Monkey H: Kwan et al., 2019). Both animals were given at least 2 weeks to recuperate from the surgery before any experiments began. Experimental protocols for afferent recordings were approved by the McGill University Animal Care Committee and were in compliance with the guidelines of the Canadian Council on Animal Care. To validate that monkey neck muscle activity exhibited similar vestibulcollic reflex (VCR) dynamics to those observed in humans, three additional male monkeys (Monkeys $\mathrm{A}, \mathrm{S}$, and $\mathrm{T}$ ) were prepared for multi-unit neck muscle recordings in the left sternocleidomastoid using indwelling electrodes identical to those described for human subject testing. Experimental protocols for the monkey neck muscle recordings were approved by the Johns Hopkins Animal Care and Use Committee, which is accredited by the Association for the Assessment and Accreditation of Laboratory and Animal Care (AAALAC).

\section{Afferent and neck muscle recordings}

During afferent recordings, monkeys were head-restrained in a primate chair mounted on top of a vestibular turntable. The left vestibular nerve was identified using the methods described by Jamali et al. (2013). Briefly, the vestibular nerve was approached via the floccular lobe of the cerebellum, characterized by its eye-movement-related activity (Lisberger and Pavelko, 1986; Cullen and Minor, 2002). Entry to the nerve was preceded by a silent period indicating that the electrode had left the cerebellum. Extracellular single-unit activity of primary semicircular canal afferents was recorded using tungsten microelectrodes (7-10 $\mathrm{M} \Omega$ and 20-25 M $\Omega$; Frederick-Haer). We limited our study to semicircular canal afferents because the objective of this study was to explore the frequency range of afferent dynamics during electrical vestibular stimulation, and because canal and otolith afferents respond similarly (Kwan et al., 2019). During neck muscle recordings, monkeys were headrestrained in a stationary primate chair. The monkeys were encouraged to turn their heads toward a treat against the head restriction to activate the left sternocleidomastoid muscle. Because this procedure led to burst of multi-unit muscle activity, it was not possible to isolate single MUs from the recorded signals.

Afferent and neck muscle signals were bandpass filtered (300-3000 $\mathrm{Hz}$ ) and digitized at $30 \mathrm{kHz}$ while the electrical stimulation signal was digitized at $1 \mathrm{kHz}$. Afferent and stimulation data were collected together on a Cerebrus Neural Signal Processor (Blackrock Microsystems).

\section{Protocol}

During afferent recordings, the monkeys remained seated and restrained throughout all experiments. Once a single afferent was isolated, the semicircular canal innervated by that primary vestibular afferent was determined based on the response to rotation along horizontal and vertical axes. To assess whether the afferents recorded in this study have similar sensitivity to previous studies, these afferents were exposed to wholebody yaw sinusoidal rotation at frequencies of $1,2,4,8,10,12$, and $16 \mathrm{~Hz}$ with peak velocity of $\sim 40 \mathrm{deg} / \mathrm{s}$. These data have previously been reported in Kwan et al. (2019). Electrical vestibular stimulation was then 
applied using the signals described in the "Electrical vestibular stimuli" section. During neck muscle recordings, the same electrical vestibular stimuli were applied once the monkeys activated their left sternocleidomastoid muscle.

\section{Data processing}

Raw single and multi-unit data from the human neck muscle recordings were first high-pass filtered using a phaseless eighth-order Butterworth digital filter $(-3 \mathrm{~dB}$ at $320 \mathrm{~Hz})$ to remove the stimulation artifact (Forbes et al., 2013). High-pass filters with large cutoffs are common for singleunit recordings (Wilkinson et al., 2008; Luu et al., 2018) because they do not affect the timing of the extracted MUs; however, their use for multiunit recordings is less common. To ensure that digital filtering did not substantially alter the characteristics of our multi-unit recordings, we examined the effect of increasing cutoffs $(120,220$, and $320 \mathrm{~Hz})$ using data from the $0-100 \mathrm{~Hz}$ stochastic electrical vestibular stimulation (see "Electrical vestibular stimuli" section). From the frequency response of multi-unit signals (sensitivity, phase, and coherence; see "Data analysis" section), we observed only a moderate decrease in sensitivity with increasing cutoff frequency, ensuring that using a cutoff of $320 \mathrm{~Hz}$ could provide useful estimates of the frequency response functions. This was further confirmed by comparing frequency responses from multi-unit EMG and single MU recordings (see Results).

Repeated trials (stochastic stimuli only; see "Electrical vestibular stimuli" section) within each subject were concatenated to create single data records for each condition and analyzed on a subject-by-subject basis. Single MU action potentials were identified and extracted using template matching software (Spike2; Cambridge Electronic Design). Single MU action potentials were identified on the basis of spatial and temporal characteristics. The algorithm designed a MU template and compared it with the MU waveform shapes to identify which action potentials belonged to a specific MU. All MU action potentials were then manually reviewed by an experienced investigator to confirm the identified units and to include action potentials unidentified by the template matching algorithm. Once MUs were sorted, firing times were exported as binary MU action potential trains to MATLAB (The MathWorks). The mean firing rate of all MUs was estimated from each single-unit recording as the inverse of the mean interspike interval (ISI; $1 / \mu_{\text {ISI }}$ ).

Primary vestibular afferents from the nonhuman primate recordings were classified as regular or irregular based on their resting discharge. Regularity of resting discharge was estimated using the coefficient of variation $(\mathrm{CV}), \mathrm{CV}=\sigma_{\mathrm{ISI}} / \mu_{\mathrm{ISI}}$, where $\mu_{\mathrm{ISI}}$ and $\sigma_{\mathrm{ISI}}$ are the mean and SD of the ISI. Because CV varies with the mean ISI, a normalized CV $\left(\mathrm{CV}^{*}\right)$ was computed using the ISI distribution to quantify resting discharge regularity following the same procedure used by Goldberg et al. (1984). Neurons with a $\mathrm{CV}^{\star}<0.1$ were classified as regular, whereas those with $\mathrm{CV}^{\star} \geq 0.1$ were classified as irregular (Sadeghi et al., 2007; Jamali et al., 2013). The mean firing rate of each neuron was also estimated as the inverse of the mean ISI $\left(1 / \mu_{\text {ISI }}\right)$.

Finally, multi-unit EMG data from the monkey neck muscle recordings were high-pass filtered using a phaseless eighth-order Butterworth digital filter $(-3 \mathrm{~dB}$ at $620 \mathrm{~Hz})$ to remove the stimulation artifact (Forbes et al., 2013). A higher cutoff was used for monkey neck muscle recordings because larger stimulation artifacts were observed compared with human data.

\section{Electrical vestibular stimuli}

Electrical vestibular stimulation was applied to both human and monkey participants using carbon rubber electrodes (human: $\sim 9 \mathrm{~cm}^{2}$; monkey: $\sim 6 \mathrm{~cm}^{2}$ ) in a binaural bipolar arrangement. The electrodes were coated with Spectra 360 electrode gel (Parker Laboratories) and secured over the participant's mastoid processes using surgical tape. To investigate the properties of vestibulocollic reflexes at frequencies observed during daily activities and transient head impact scenarios (Carriot et al., 2014; Wu et al., 2016, 2017), we applied sinusoidal and stochastic signals up to $300 \mathrm{~Hz}$ during both human and animal testing. Specifically, transient head impacts produce acceleration profiles resembling raised cosines with periods as low as $\sim 0.003$ s (i.e., $\sim 300 \mathrm{~Hz}$ ) (Wu et al., 2016). Sinusoidal signals ensured that the stimulus power at individual frequencies would be sufficient to elicit responses in neck MUs and vestibular afferents. Stochastic signals, which distribute signal power across all frequencies, allowed us to examine frequency responses between the isolated frequencies tested with the sinusoidal signals across the entire bandwidth $(0-300$ $\mathrm{Hz}$ ).

During human volunteer experiments, the stimuli were delivered as analog signals via a data acquisition board (20 kHz; PXI-6289; National Instruments) to an isolated constant-current stimulator (STMISOLA; Biopac). All subjects were exposed to the same realizations of the following current stimuli: 1) $80 \mathrm{~s}$ sinusoidal stimuli at frequencies of 25, 50, 75, $100,150,200,250$, and $300 \mathrm{~Hz}$ with a peak amplitude of $\pm 3 \mathrm{~mA}$, and 2) $80 \mathrm{~s}$ stochastic vestibular stimuli with bandwidths of $0-100$ and $0-300$ $\mathrm{Hz}$ and RMS currents of $\sim 1.70 \mathrm{~mA}$ (peak amplitudes of $\pm 5 \mathrm{~mA}$ ). All signals were generated off-line with MATLAB. Single sine waves were delivered once and stochastic signals twice for both the single and multiunit recording blocks (see "Protocol" section). The $0-100 \mathrm{~Hz}$ stochastic stimulus was used to evaluate the effects of our filtering approach as mentioned above and matched previous studies characterizing frequency responses in human neck muscles (Forbes et al., 2013, 2014, 2018).

During animal experiments, the stimuli were generated using MATLAB and delivered as analog signals to a constant-current stimulator (STMISOLA; Biopac) via a QNX-based real-time data acquisition system (Hayes et al., 1982) or an arbitrary waveform generator (Keysight Technologies) as has been previously described (Kwan et al., 2019). The animals were exposed to the following current stimuli: 1) sinusoidal stimuli at frequencies of $1,2,4,8,16,25,50,75,100,150,200,250$, and $300 \mathrm{~Hz}$ at a peak amplitude of $\pm 1 \mathrm{~mA}$, and 2) $60 \mathrm{~s}$ stochastic stimulation with bandwidths of $0-100$ and $0-300 \mathrm{~Hz}$ and RMS current of 0.43 and $0.23 \mathrm{~mA}$, respectively (peak amplitudes of $\pm 1 \mathrm{~mA}$ ). The low-frequency stimuli $(1-25 \mathrm{~Hz})$ were used for comparison to previously recorded af ferents (Kwan et al., 2019). We collected afferent data for $\geq 10$ cycles for lower frequency stimuli $(1-25 \mathrm{~Hz})$ and $>500$ cycles for high-frequency stimuli $(50-300 \mathrm{~Hz})$. For monkey neck muscle responses, preliminary testing indicated that the stochastic stimuli did not evoke sufficient neck muscle activity to estimate vestibulocollic reflex dynamics. Therefore, we have limited the reporting of these data to single sine responses identical to those used in our human experiments.

\section{Data analysis}

We first examined the firing rates of human neck MUs and monkey primary vestibular afferents evoked by the sinusoidal electrical stimuli by plotting cycle histograms. Firing rates were visualized by dividing each cycle into 16 equal-duration bins, calculating the average number of spikes in each bin across repeated stimulus cycles and dividing by the bin size. These results were used to examine the average modulation of MU and primary afferent firing rates on a unit-by-unit basis throughout the stimulus cycle. For the single MU data, the number of cycles contributing depended on the baseline firing rate of the unit but varied between $\sim 1200-3000$ cycles for each subject in each trial. For the afferent data, the number of stimulus cycles contributing to the histograms was between 10 and 20 cycles for lower frequency stimuli $(1-25 \mathrm{~Hz})$ and $>480$ cycles for high-frequency stimuli $(50-300 \mathrm{~Hz})$.

To estimate the frequencies at which neck MUs (single MU measures from humans and multi-unit measures from humans and monkeys) and vestibular primary afferents exhibit linear responses to electrical vestibular stimuli, we first estimated the coherence between the input electrical vestibular stimulus $\mathrm{s}(t)$ and the binary spike train (or rectified multi-unit EMG signal) $r(t)$ during both sinusoidal and stochastic stimulation (Rosenberg et al., 1989; Halliday et al., 1995). Coherence is a measure of the linear relationship of the evoked response to the stimulus at a frequency $f$ and is given by $C(f)=\left|P_{s r}(f)\right|^{2} /\left(P_{s s}(f) P_{r r}(f)\right)$ where $P_{s r}(f)$ is the stimulus-response cross spectrum, $P_{s s}(f)$ is the power spectrum of the stimulus, and $P_{r r}(f)$ is the power spectrum of the spike train (or rectified multi-unit EMG signal). Coherence was estimated using the stimulus cycle window for sinusoidal stimuli or $1 \mathrm{~s}$ windows (frequency resolution of $1 \mathrm{~Hz}$ ) for stochastic stimuli within each single MU, multiunit EMG or afferent recording. Spectral estimates obtained for human and animal data during stochastic stimulation were computed using 
multitaper estimation techniques with eight Slepian functions (Jarvis and Mitra, 2001) as implemented previously (Sadeghi et al., 2007). At each frequency point, coherence ranges from 0 for systems with no linear relation to 1 for linear systems without noise (Pintelon and Schoukens, 2012). The $95 \%$ confidence limit for coherence spectra was derived from the number of disjoint segments to indicate frequencies where coherence was significantly different from 0 (Halliday et al., 1995). Coherence typically decreased, and eventually remained below the confidence limit, with increasing frequencies. We estimated the bandwidth of neck MU (single MU and multi-unit EMG) and afferent responses during stochastic stimulation using the mean responses across subjects or afferents when coherence fell below the $95 \%$ confidence limit for a frequency range of at least $5 \mathrm{~Hz}$.

To then characterize the linear component of responses from neck motoneuron (single MU and multi-unit EMG) and vestibular primary afferent activity across the stimulated frequencies, we estimated the mean sensitivity- and phase-frequency responses from the transfer function $H(f)=P_{s r}(f) P_{s s}(f)$. Sensitivity and phase indicate the magnitude and timing of the output neural responses (single MU, multi-unit EMG, or vestibular afferent) relative to the input electrical stimulus at frequencies with significant coherence. Coherence values below the $95 \%$ confidence limit indicates a lack of a linear association between the input and output signal, making the sensitivity and phase estimates unreliable (Halliday et al., 1995). When this occurred, sensitivity and phase estimates for that single MU, multi-unit EMG or vestibular afferent signal at that frequency were removed from the group average and 95\% confidence interval. Because monkeys did not maintain continuous neck muscle activity throughout the entire duration of each trial, we only used segments of data where sufficient muscle activity was detected. When the maximum of the high-pass filtered (see above), rectified EMG signal over a $1 \mathrm{~s}$ window fell below the RMS of the entire filtered signal, that window was removed from the contribution to the gain and phase estimates. This resulted in trials ranging from 62 to $80 \mathrm{~s}$ of data used to estimate the sensitivity, phase and coherence.

To determine whether human multi-unit EMG responses could be used to extract the frequency response of human single neck MUs to electrical vestibular stimulation, we compared the sensitivity and phase responses during sinusoidal stimulation using a linear mixed model (fixed effects: measurement type and frequency; random effect; subject). Note that single MU sensitivities were first normalized to multi-unit EMG responses at $25 \mathrm{~Hz}$ due to differing units between single-MU and multi-unit EMG recordings (spk/s/mA vs $\mathrm{uV} / \mathrm{mA}$ ). We then determined whether the sensitivity and phase differed between regular and irregular primary afferents and with increasing frequency during sinusoidal stimulation using a linear mixed model (fixed effects: afferent type and frequency; random effect: afferent number) as previously assessed during low-frequency stimulation (0-25 Hz; Kwan et al., 2019). We were primarily interested in the difference between regular and irregular afferents. Therefore, when interactions were observed, we decomposed them to determine the effect of afferent type at each frequency using Bonferroni corrected pairwise comparisons. Additional Bonferroni corrected pairwise comparisons were made to assess the effect of stimulus frequency for both afferent types. To determine whether similar features in the vestibulocollic response dynamics were observed in both species, we also examined the sensitivity and phase of the monkey multi-unit EMG. Estimated sensitivities were normalized to the mean sensitivity measured across all frequencies.

Finally, to quantify how likely human neck MUs and monkey vestibular afferents fire at fixed time points within the cycle of each sinusoidal frequency, we estimated a phase-locking index (PLI) using the entropy of the cycle histogram (Kajikawa and Hackett, 2005) given by the following:

$$
\begin{gathered}
P L I=1-\frac{E_{0}}{E_{\max }} \\
E_{0}=-\left\langle P(\phi) \log _{2} P(\phi)\right\rangle \\
E_{\max }=\log _{2} N
\end{gathered}
$$

where $P(\phi)$ is the probability of firing a spike as a function of stimulus phase, $E_{0}$ is the entropy of the probability distribution, and $E_{\max }$ is the maximum entropy possible (that of a uniform distribution). This measure quantifies the degree of phase-locking and ranges between 0 and 1 , where 1 indicates fixed timing of neural firing within the stimulus cycle. Since phase-locking is a nonlinear behavior, this also provided an assessment of any nonlinear distortions on the linear estimates of sensitivity, phase and coherence. Because PLI is sensitive to the number of cycles used in its estimate, we limited our analysis to 10 cycles for low-frequency sinusoidal stimuli $(1-25 \mathrm{~Hz})$ and 20 cycles for high-frequency sinusoidal stimuli $(50-300 \mathrm{~Hz})$. Importantly, at frequencies above the resting firing rate of the MUs and vestibular afferents many cycles of the stimulus are missed. Therefore, cycles without spikes were skipped when constructing the 10 or 20 cycles. The number of cycles used in the estimate was increased to 50 for neck MUs (again skipping cycles without spikes) because of their lower firing rate $(\sim 15$ spikes/s) compared with vestibular afferents $(\sim 100$ spikes/s). We observed high variability in the PLI estimates for the $25-300 \mathrm{~Hz}$ frequencies due to the low number of spikes within each cycle (often only 1 or 2) for both neck MUs and vestibular afferents. For these higher frequencies, we therefore used a resampling approach by repeating our analysis 20 times using windows chosen randomly across our time series and took the mean as the final estimate of PLI for each MU or vestibular afferent. Finally, to determine any changes in phase-locking for single MUs with increasing frequency, we analyzed the PLI using a linear mixed model (fixed effect: frequency; random effect; subject). Similarly, we assessed any differences in phase-locking between regular and irregular primary vestibular afferents and with increasing frequency by analyzing PLIs using a linear mixed model (fixed effect: afferent type and frequency; random effect: afferent number).

\section{Statistics}

A significance level of 0.05 was used for all analyses and all values are expressed as mean \pm SEM. All statistical analyses were performed using SPSS software (IBM).

\section{Results}

\section{Human neck MU responses}

To characterize the frequency bandwidth of the electricallyevoked vestibulocollic reflexes in humans (Fig. 1), we first evaluated the single $\mathrm{MU}$ and multi-unit EMG responses from the sternocleidomastoid muscle. We focused our analysis on the sternocleidomastoid muscle because it displays consistent responses to the electrical stimulus at high frequencies (Forbes et al., 2013, 2014). Subjects maintained neck muscle activity through a flexion contraction while sinusoidal and stochastic electrical vestibular stimuli were applied (see Materials and Methods). During multi-unit EMG trials, subjects maintained constant muscle activity (i.e., RMS of filtered EMG) that did not vary across trials $\left(p=0.729, F_{(9,63)}=0.68\right)$. During single MU trials, subjects maintained a mean firing rate of $11.1 \pm 1.0 \mathrm{spk} / \mathrm{s}(n=8)$, well below the resting firing rate of vestibular afferents (see below) and the lowest frequency sinusoidal stimulus $(25 \mathrm{~Hz})$. We then examined the response characteristics of single MU and multiunit EMG recordings to sinusoidal and stochastic stimulation. Although these two stimuli were expected to produce similar responses at overlapping frequencies, sinusoidal signals are more likely to elicit responses in neck MUs at higher frequencies even iflow-pass filtering progressively diminishes the evoked muscle responses due to their higher power at the given stimulus frequency. Stochastic signals, on the other hand, which distribute power across all frequencies, provide a detailed assessment of responses between and across all of the isolated sinusoidal frequencies.

Figure $2 A$ presents the raster plots and firing rate histograms of example single MU data, as well as a time series of multi-unit EMG data, evoked by 50,100, and $200 \mathrm{~Hz}$ sinusoidal stimuli. Both the single MU firing and multi-unit EMG data were mod- 
A
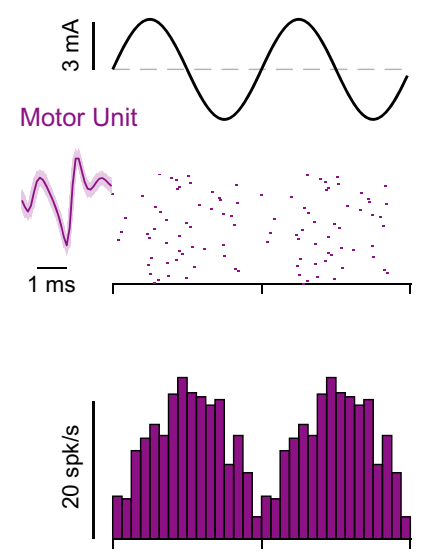

EMG

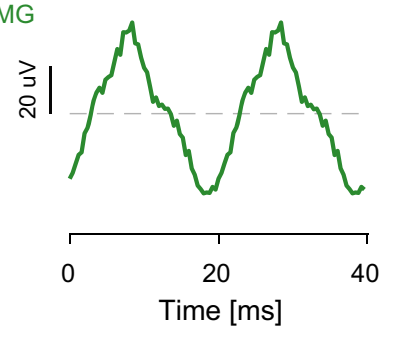

B Sensitivity [spks/s/mA or uV/mA]

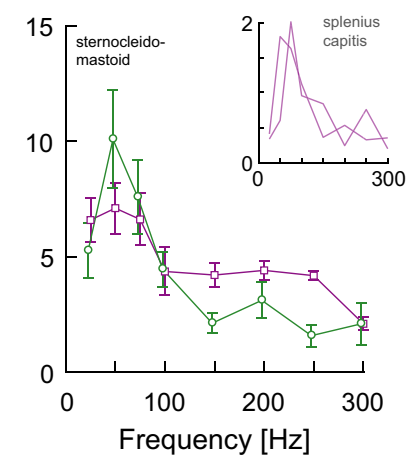

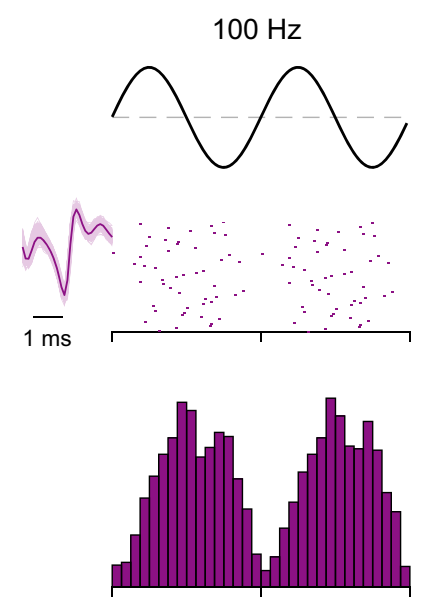
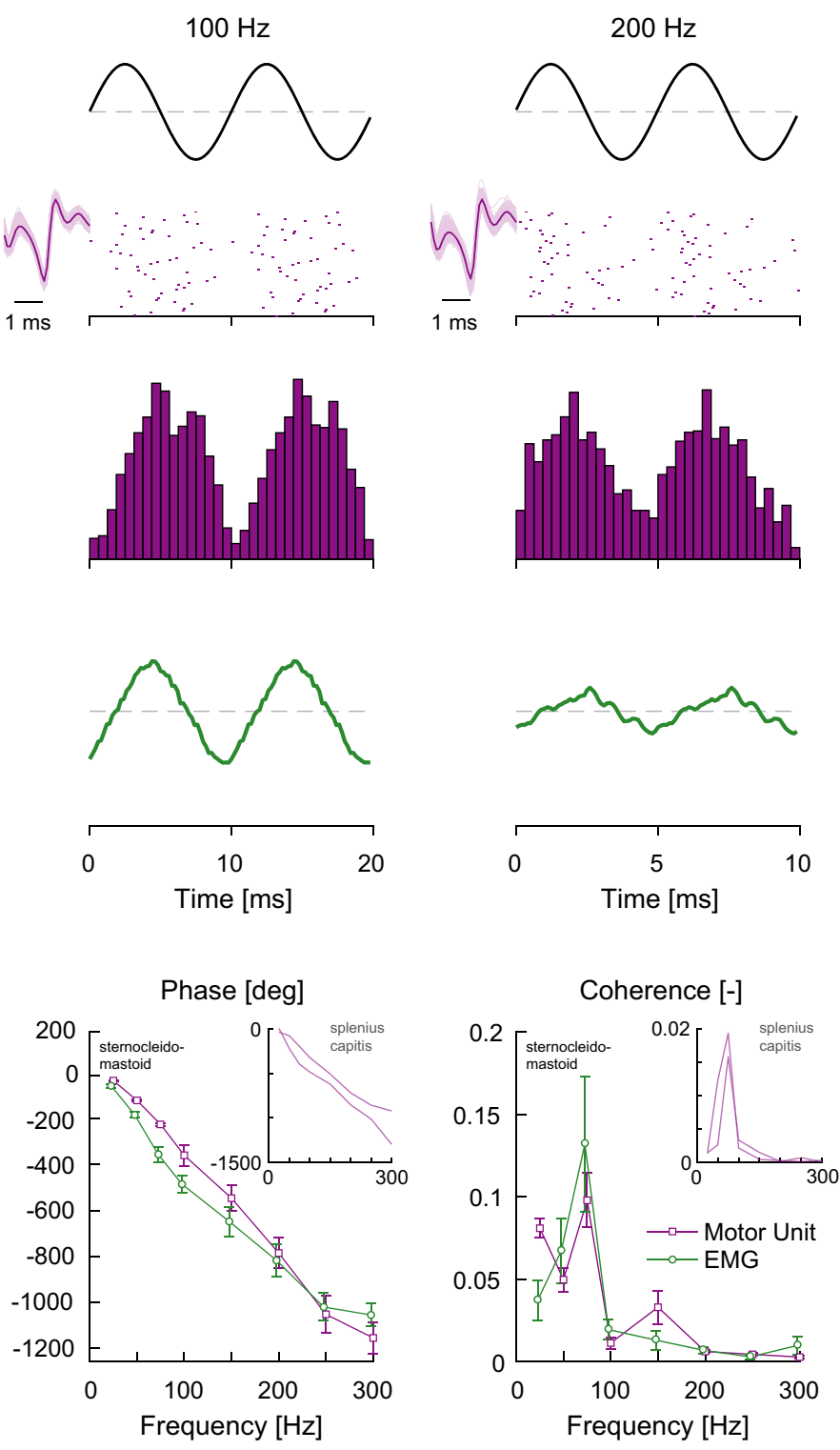

Figure 2. Firing behavior and response dynamics of human sternocleidomastoid muscle single MUs to high-frequency $(25-300 \mathrm{~Hz})$ sinusoidal electrical vestibular stimulation. $A$, Raster plots from 50 cycles of stimulus oscillation and mean firing rate histograms of example neck MUs together with multi-unit EMG responses during 50,100 and $200 \mathrm{~Hz}$ stimuli. Insets next to the raster plots are the result of the template match for the identified MU in the recording. $\boldsymbol{B}$, Population average sensitivity, phase and coherence plotted as a function of frequency for single-unit (purple, $n=8$ ) and multi-unit EMG (green, $n=8$ ) responses from sternocleidomastoid muscle activity. Error bars indicate 1 SEM. Sensitivity, phase, and coherence of human splenius capitis muscle single MUs $(n=2)$ are included as insets for comparison.

ulated by the input stimulus. These responses were consistent across our dataset. Mean coherence in both single MU $(n=8)$ and multi-unit EMG responses $(n=8)$ was above the $95 \%$ confidence interval for all sinusoidal frequencies (Fig. 2B). Furthermore, for both single MUs and multi-unit EMG, sensitivities increased similarly $\left(p=0.814, F_{(1,86.1)}=0.1\right.$; normalized sensitivities) over the $25-75 \mathrm{~Hz}$ frequency range before falling to a plateau at higher frequencies $(>100-150 \mathrm{~Hz})$. Generally, phases decreased with increasing frequencies for both single MU and multiunit EMG measures, and both measures decreased at a monotonic rate with increasing frequency consistent with an $\sim 10-13$ ms delay relative to the input. Phases, however, differed across single MU and multi-unit EMG measures $\left(~ p=0.032, F_{(1,100.0)}=4.7\right)$, with pairwise comparisons revealing that multi-unit EMG phase lags were $\sim 60-$ $130^{\circ}$ lower at only 50 and $75 \mathrm{~Hz}(p<0.008$ and $p=0.030$, respectively, Bonferroni correction). Recordings from the right splenius capitis muscle in two additional subjects revealed similar sensitivity and phase responses to the electrical vestibular stimuli relative to the sternocleidomastoid muscle (Fig. $2 B$, insets). Taken together, these results suggest that (1) the bandwidth of vestibular-evoked neck muscle responses extends as high as $300 \mathrm{~Hz}$ and (2) that the frequency response of the vestibulocollic reflex estimated with multiunit EMG reflects the response of neck MU activity to vestibular stimuli.

To provide a more detailed assessment of vestibular-evoked neck muscle response dynamics, we next characterized single-MU and multi-unit EMG responses to stochastic vestibular stimulation. Figure 3 illustrates the coherence, sensitivity and phase responses during stochastic stimuli with data from sinusoidal stimuli (Fig. 2B) superimposed for comparison. We observed that mean coherence between the stochastic stimuli and neck muscle activity was only significant up to $\sim 120 \mathrm{~Hz}$ for the single 

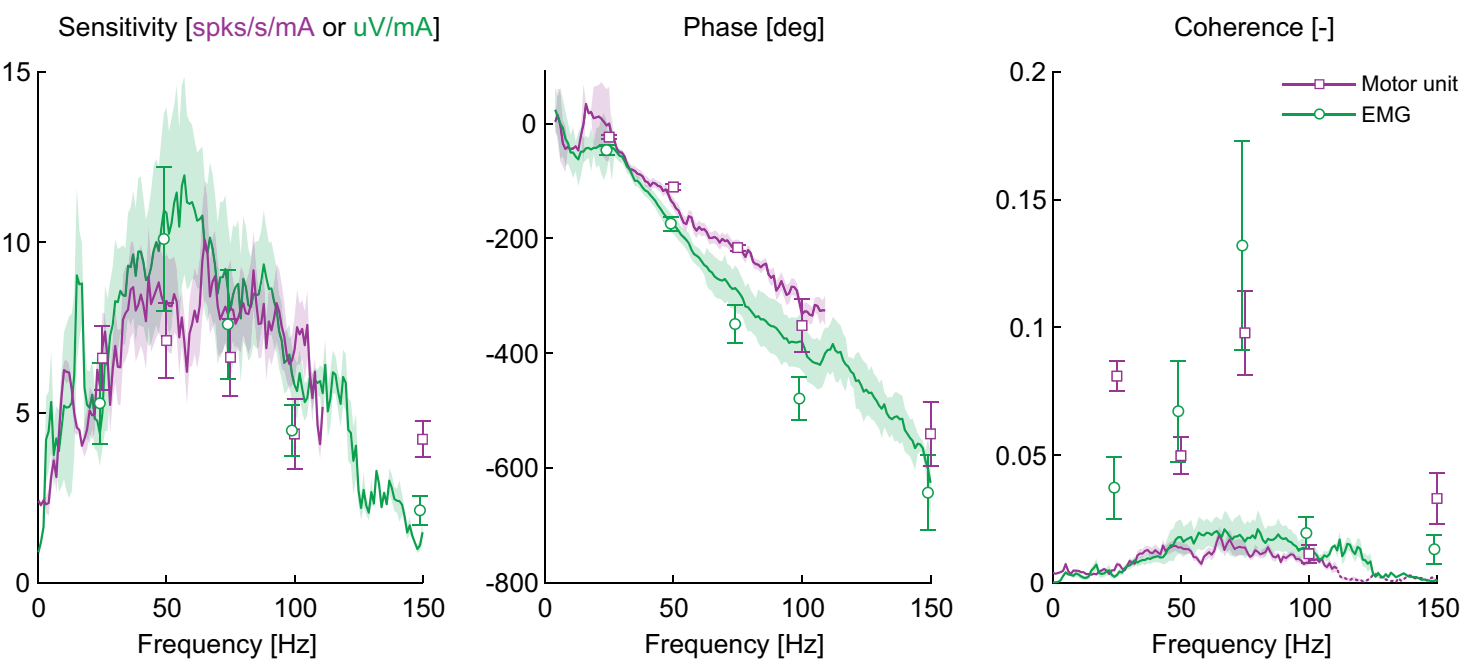

Figure 3. Response dynamics of human sternocleidomastoid muscle single MUs (purple, $n=8$ ) and multi-unit EMG (green $n=8$ ) during sinusoidal (square and circle symbols) and stochastic (lines) vestibular stimulation. Population averages of sensitivity, phase and coherence demonstrate a similarity in responses across the two stimuli. Sensitivity peaked at $75-85 \mathrm{~Hz}$ and decreased thereafter, whereas phase primarily decreased with frequency. Single MU sensitivity and phase observed in response to stochastic stimuli were plotted up to $\sim 120 \mathrm{~Hz}$ because mean coherence fell below the $95 \%$ confidence interval (dotted line segment in coherence plot at frequencies $>120 \mathrm{~Hz}$ ). Error bars and shaded regions indicate $1 \mathrm{SEM}$ for responses to single sines and stochastic stimulation, respectively.

MU recordings and $\sim 150 \mathrm{~Hz}$ for the multi-unit EMG measurements (Fig. 3, dotted line segment in right panel for single MU measures at frequencies $>120 \mathrm{~Hz}$ ); as a result, sensitivity and phase estimates were only plotted where significant coherence was observed. While this reduced bandwidth differs from the higher frequency (up to $300 \mathrm{~Hz}$ ) neck muscle responses during sinusoidal stimuli, it was likely due to the distributed power among all frequencies included in the stochastic stimulus. Indeed, where coherence was significant, sensitivity and phase for single MUs and multi-unit EMG showed similar trends as those evoked by sinusoidal stimuli, whereby sensitivity peaked between 50 and $75 \mathrm{~Hz}$ and phase progressively decreased with increasing frequency. Altogether, our human data show that neck muscles respond to electrical vestibular stimulation at frequencies associated with head-impact (i.e., up to $300 \mathrm{~Hz}$ ) with sensitivities that remain equivalent to or higher than those at frequencies typically associated with normal head movements (i.e., $0-30 \mathrm{~Hz}$ ).

\section{Primary vestibular afferent responses}

Having shown that human neck muscles respond to stimulation at frequencies up to those reported during head impacts (Wu et al., 2016), we next sought to identify the vestibular afferent origins of the evoked motor responses. Specifically, to understand the relationship between activation of the vestibular system and the neck responses, we recorded from single isolated vestibular afferents in monkeys during the same stimulation conditions (Fig. 4A). We first recorded the spiking activity of 26 semicircular canal afferents, and assessed the regularity of their resting firing rate (see Materials and Methods). $n=15$ afferents were classified as regular $\left(\mathrm{CV}^{*}=0.05 \pm 0.01\right)$ and $n=12$ afferents were classified as irregular $\left(\mathrm{CV}^{\star}=0.29 \pm 0.05\right)$. The mean resting firing rates were $108 \pm 6 \mathrm{spk} / \mathrm{s}$ for regular afferents and $73 \pm 12 \mathrm{spk} / \mathrm{s}$ for irregular afferents. We then examined the response characteristics of these afferents during sinusoidal electrical stimulation over frequencies commonly used to assess vestibular responses during motion and electrical stimuli (1-25 Hz). Consistent with previous reports (Kwan et al., 2019), the input stimulus modulated the firing rate of vestibular afferents across sinusoidal frequencies at $1-25 \mathrm{~Hz}$ (Fig. $4 B$, insets). The sensitivity of individual irregular afferents to the input stimulus was higher than all regular afferents at all frequencies, and the sensitivity of both afferent types increased as a function of frequency. Analysis of the group data confirmed these observations (Fig. $4 B$, insets): significant main effects were observed for afferent type $\left(p<0.001, F_{(1,25.2)}=\right.$ $39.5)$ and frequency $\left(p<0.001, F_{(5,95.0)}=25.0\right)$, as well as a significant interaction of afferent type $\times$ frequency $(p<0.001$, $\left.F_{(5,95.0)}=5.2\right)$. Pairwise comparisons confirmed the higher sensitivity of irregular afferents $(\sim 2-4$ times) at all frequencies (multiple $p<0.01$, Bonferroni correction). In contrast, phase leads of both regular and irregular afferents to these lower frequency stimuli $(0-25 \mathrm{~Hz})$ were similar $\left(p=0.833, F_{(1,26.1)}=0.1\right)$ and both increased as a function of frequency $\left(p<0.001, F_{(5,99.5)}\right.$ $=19.8)$, with no afferent type $\times$ frequency interaction $(p=$ $\left.0.852, F_{(5,99.5)}=0.4\right)$

To establish how afferents respond to high-frequency electrical stimulation (i.e., $>25 \mathrm{~Hz}$ ), we examined the response characteristics in the same group of neurons during sinusoidal stimuli up to $300 \mathrm{~Hz}$. Figure $4 A$ shows the raster plots and firing rate histograms of example regular and irregular afferent responses evoked by 50, 100 and $200 \mathrm{~Hz}$ sinusoidal electrical vestibular stimuli. The evoked activity of individual afferents at high frequencies was consistent with low-frequency afferent responses to electrical stimulation: (1) firing rates of both afferent types modulated with the input stimulus, and (2) irregular afferents had a greater sensitivity compared with regular afferents. These results were confirmed across our dataset for all afferents. Mean coherence was above the $95 \%$ confidence limit at all frequencies for both irregular and regular afferents (Fig. 4B). For response sensitivities, significant main effects were observed for afferent type $\left(p<0.001, F_{(1,23.1)}=28.1\right)$ and frequency $\left(p<0.001, F_{(12,231.8)}\right.$ $=33.1)$, as well as a significant interaction of afferent type $X$ frequency $\left(p<0.001, F_{(12,231.8)}=3.4\right)$. Decomposition of the interaction revealed that, except for 100 and $300 \mathrm{~Hz}$ stimuli, sensitivity for the irregular afferents was $\sim 2-4$ times higher than regular afferents (Fig. $4 B)(p<0.05$ for all frequencies except 100 and $300 \mathrm{~Hz}$, Bonferroni correction). For frequencies $>100 \mathrm{~Hz}$, however, sensitivities in both afferent types plateaued (Fig. 4B) and no effect of frequency was observed from 150 to $300 \mathrm{~Hz}$ (all 
A

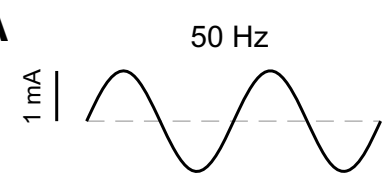

regular
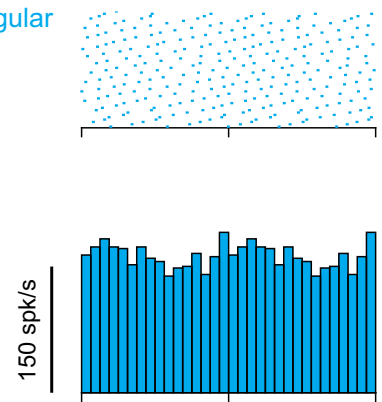

irregular
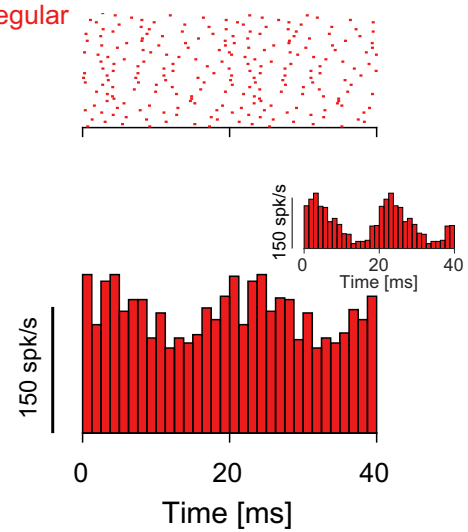

B

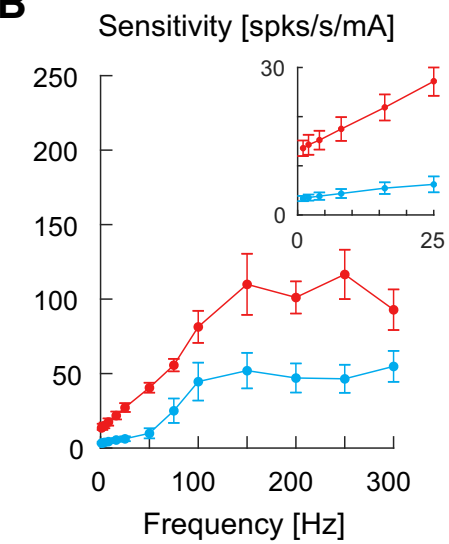

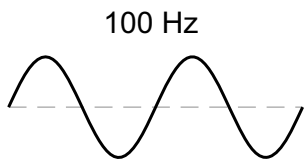
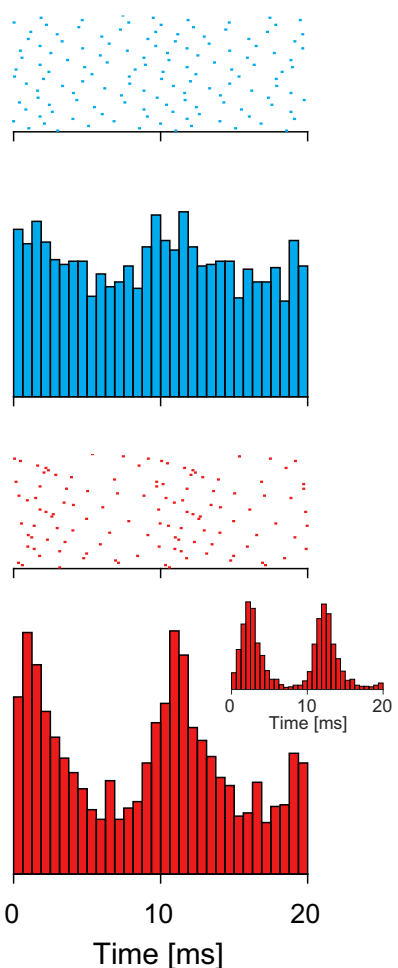

Time $[\mathrm{ms}]$

Phase [deg]

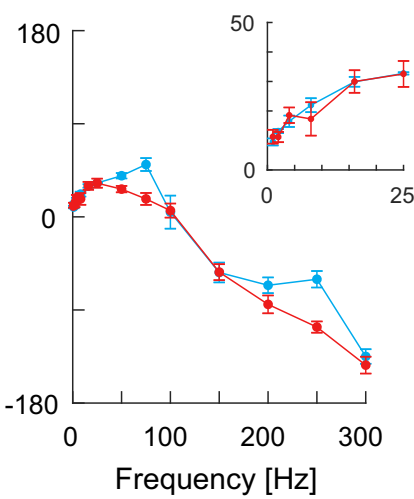

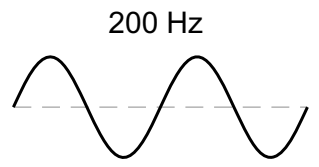
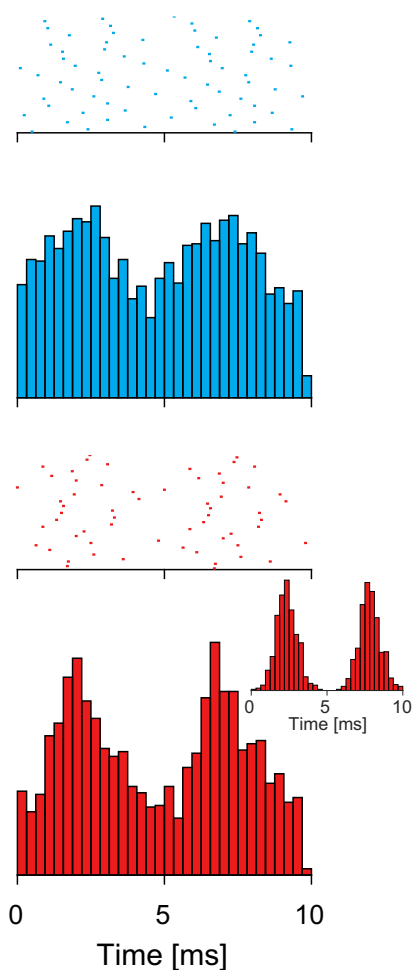

Coherence [-]

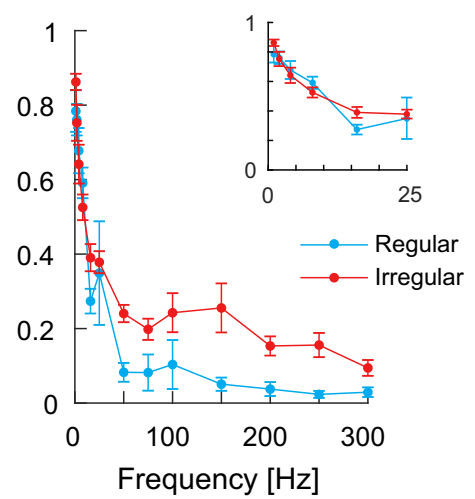

Figure 4. Firing behavior and response dynamics of monkey vestibular afferents to high-frequency $(0-300 \mathrm{~Hz})$ sinusoidal electrical vestibular stimulation. $A$, Raster plots from $50 \mathrm{cycles}$ of stimulus oscillation and mean firing rate histograms of example regular (blue) and irregular (red) afferents at 50, 100, and $200 \mathrm{~Hz}$ stimuli. The majority of afferents responded to the electrical stimulus by modulating their firing rate, however, some irregular vestibular afferents tended to lock their firing to specific phases of the input stimulus (insets), particularly when the stimulus frequency exceeded $\sim 100 \mathrm{~Hz}$. B. Population average sensitivity, phase and coherence plotted as a function of frequency for regular (blue, $n=10-15$ ) and irregular (red, $n=8-12$ ) afferents. Responses at low frequencies are included as insets for comparison. Error bars indicate 1 SEM.

pairwise comparisons $p>0.05$ ). Phase responses at high frequencies $(>25 \mathrm{~Hz})$ in both afferents also differed from low frequencies: phase leads continued to increase reaching $\sim 40^{\circ}$ at $50-75$ $\mathrm{Hz}$, however, at frequencies $>75 \mathrm{~Hz}$ the phase began lagging the input stimulus and reached $\sim-135^{\circ}$ at $300 \mathrm{~Hz}$. Significant main effects were observed for afferent type ( $\left.p=0.003, F_{(1,19.5)}=11.5\right)$ and frequency $\left(p<0.001, F_{(12,232.9)}=129.5\right)$, as well as a significant interaction of afferent type $\times$ frequency $(p=0.002$, $\left.F_{(12,232.9)}=2.7\right)$. Decomposition of the interaction revealed that phase between the two afferent types differed only at $75 \mathrm{~Hz}$ ( $p=$ $0.009)$ and $250 \mathrm{~Hz}(p=0.003)$. At these two frequencies, however, the mean differences in phase $\left(75 \mathrm{~Hz}: 33.3 \pm 8.5^{\circ} ; 250 \mathrm{~Hz}\right.$ : $46.2 \pm 9.7^{\circ}$ ) represent a time difference of 1.2 and $0.5 \mathrm{~ms}$, respectively, in their response to the electrical input stimulus. Overall, these results demonstrate that afferents are capable of responding to high frequency (up to $300 \mathrm{~Hz}$ ) transmastoid electrical stimuli.

We next examined the sensitivity- and phase-frequency estimates during stochastic vestibular stimulation in a second group 
A

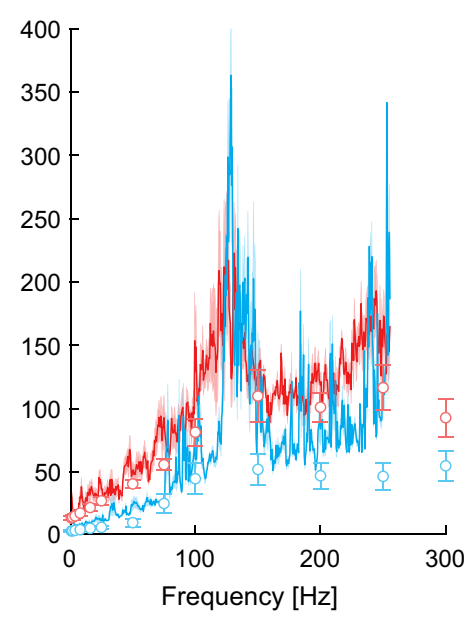

B

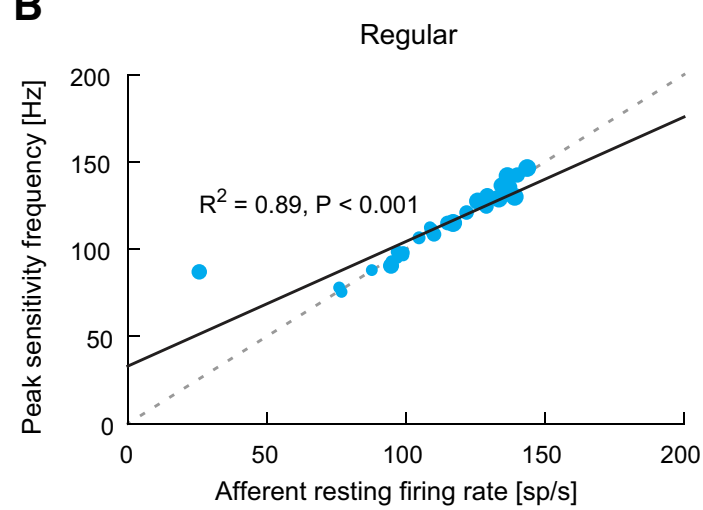

Phase [deg]

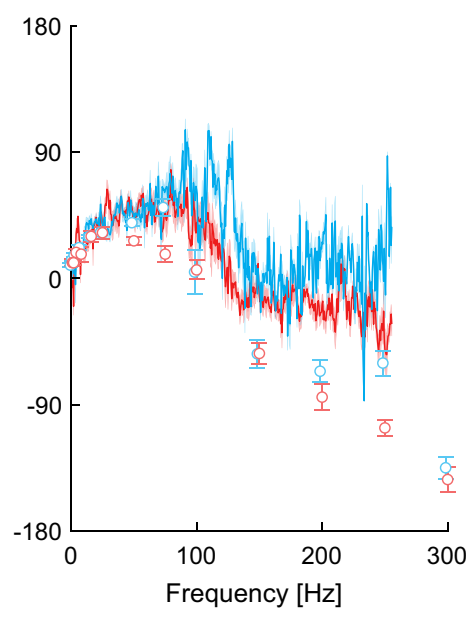

C

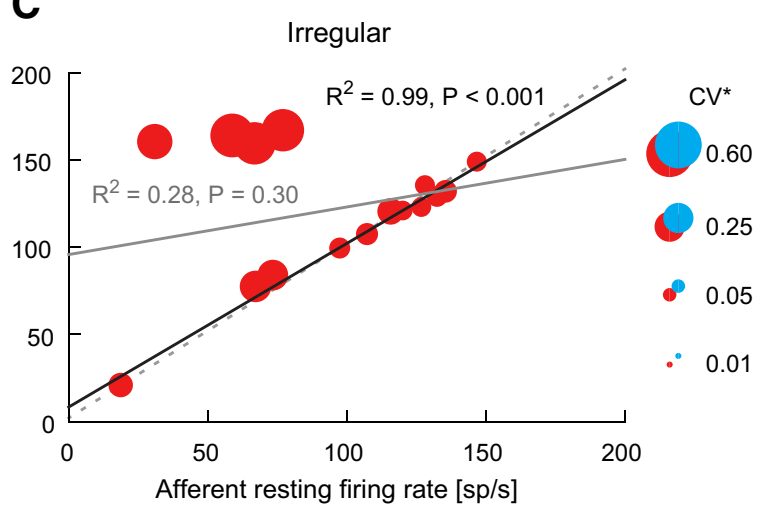

Figure 5. Frequency responses of monkey vestibular afferents. $A$, Vestibular afferent response dynamics during sinusoidal (see circles; regular: $n=10-15$; irregular: $n=8-12$ ) and stochastic (see lines; regular: $n=31$; irregular: $n=16$ ) vestibular stimulation. Sensitivity and phase observed in response to stochastic stimuli were plotted up to $\sim 250 \mathrm{~Hz}$ because mean coherence fell below the $95 \%$ confidence interval (dotted line segments in stochastic coherence plot at frequencies $>250 \mathrm{~Hz}$ ). For clarity, coherence spectra from sine and stochastic stimuli are plotted separately. Error bars and shaded regions represent 1 SEM for responses to single sines and stochastic stimulation, respectively. $\boldsymbol{B}$, Peak sensitivity frequency vs afferent resting firing rate for regular afferents $(n=$ 31). The solid black line is the linear fit. C, Peak sensitivity frequency versus afferent resting firing rate for irregular afferents $(n=16)$. The solid gray line is the linear fit with all afferents, while the solid black line is the linear fit when afferents with $\mathrm{CV}^{*}>0.3$ were removed from the analysis. For both $\boldsymbol{B}$ and $\boldsymbol{C}$, marker size (area) depicts the $\mathrm{CV}^{*}$ of each afferent according to the size scale and the dotted gray lines depict the unity relationship.

of afferents (regular, $n=31$; irregular, $n=16$ ) and compared their responses to those evoked during sinusoidal vestibular stimuli (Fig. $5 A$ ). The $\mathrm{CV}^{*}$ (regular: $0.06 \pm 0.01$, irregular: $0.23 \pm$ 0.04 ) and mean resting firing rates (regular: $111 \pm 4 \mathrm{spk} / \mathrm{s}$, irregular: $94 \pm 10 \mathrm{spk} / \mathrm{s}$ ) of these afferents were comparable to those recorded during sinusoidal vestibular stimuli (Student's $t$ test: regular both $p>0.05, t_{(44)}<0.87$; irregular both $p>0.05, t_{(27)}<$ 1.4). Primary vestibular afferent activity evoked by the stochastic stimuli produced significant responses spanning a frequency range from 0 to $250 \mathrm{~Hz}$. Comparison with responses to sinusoidal stimuli over this range revealed similar general trends, namely increasing sensitivity and phase lead up to $\sim 75 \mathrm{~Hz}$, as well as a plateau in sensitivity and decreasing phase above $100 \mathrm{~Hz}$ for both afferent types. Differences in phase at high frequencies $(>100$ $\mathrm{Hz}$ ), however, were observed between stochastic and sinusoidal stimuli (see nonoverlapping confidence intervals Fig. 5A, middle). Above $100 \mathrm{~Hz}$, the phase between afferent activity and stochastic vestibular stimuli varied but remained around $\sim 0^{\circ}$, while the phase between afferent responses and sinusoidal stimuli reached lags up to $\sim-135^{\circ}$ at $300 \mathrm{~Hz}$. More notably, stochastic electrical stimuli elicited substantial peaks in sensitivity at frequencies between 100 and $150 \mathrm{~Hz}$ in both regular and irregular afferents (but more defined in regular afferents) that were not observed in the mean response to sinusoidal stimuli (Fig. 5A). On average, these sensitivity peaks were $\sim 8$ and 2 times higher than the sensitivity at surrounding frequencies during sinusoidal stimulation $(100$ and $150 \mathrm{~Hz})$ for regular and irregular afferents, respectively. These peaks also occurred on average at frequencies of $112 \pm 20 \mathrm{~Hz}$ for regular afferents and $121 \pm 38 \mathrm{~Hz}$ for irregular afferents, which, for the regular afferents, was similar to their resting firing rate $(111 \pm 4 \mathrm{spk} / \mathrm{s})$. The frequency at which sensitivity peaked also aligned with the shift in phase where afferent firing rate began to lag the stimulus. These results suggest, at least for regular afferents, that the frequency of peak sensitivity is linked to the afferent resting discharge regularity.

To test this possibility, we plotted the frequency at which peak sensitivity was identified against the resting firing rate of each afferent together with its $\mathrm{CV}^{\star}$ (with $\mathrm{CV}^{\star}$ depicted as symbol size) (Fig. $5 B, C$ ). As shown in Figure $5 B$, we found a strong positive linear correlation for regular afferents $\left(R^{2}=0.89, p<0.001\right.$; solid black line), which with the exception of a single afferent was characterized by a unity relationship (dotted gray line) between the frequency of peak sensitivity and resting firing rate. Figure $5 C$ illustrates this same relationship plotted for irregular afferents. Although there was no significant correlation between the frequency of peak sensitivity and resting firing across all irregular 

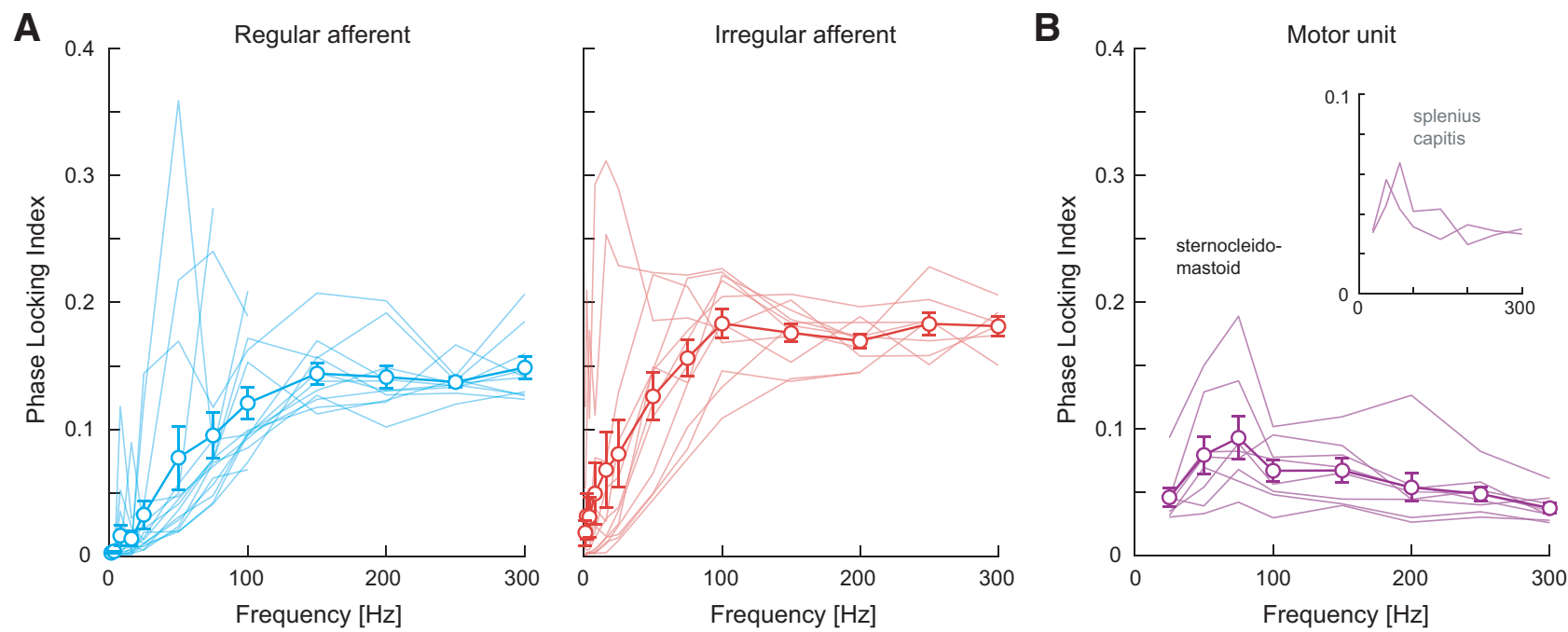

Figure 6. Phase-locking index of monkey single vestibular afferents $(\boldsymbol{A})$ and human sternocleidomastoid muscle single MUs $(\boldsymbol{B})$ plotted as a function of frequency. $\boldsymbol{A}$, Phase-locking indices estimated for regular (blue) and irregular (red) vestibular afferents increased to a plateau at $\sim 150$ and $\sim 100 \mathrm{~Hz}$, respectively. Error bars indicate 1 SEM (regular: $n=10-15$; irregular: $n=8-12$ ). $B$, Phase-locking index estimated for MUs reached a peak of $\sim 0.1$ at $75 \mathrm{~Hz}$ after which it progressively decreased with frequency. Error bars indicate 1 SEM $(n=8)$. Phase-locking index of human splenius capitis muscle single MUs $(n=2)$ are included as insets for comparison.

afferents $\left(R^{2}=0.28, p=0.30\right.$; solid gray line), there was a positive linear relationship $\left(R^{2}=0.99, p<0.001\right.$; solid black line $)$ when we considered neurons with lower coefficients of variation (i.e., $\mathrm{CV}^{\star}<0.3: n=12$ ). Specifically, when we excluded the 4 afferents with the highest resting rate variability (i.e., $n=4$; largest circles), the frequency of peak sensitivity and resting firing was again described by a unity relationship (cf. dotted gray lines in Fig. $5 B, C$ ). Further, the sensitivity of the four excluded, highly irregular, afferents did not display distinct peaks across the stimulated bandwidth but rather progressively increased to a plateau around $100-150 \mathrm{~Hz}$. Thus, taken together our results suggest that the frequency of a given afferent's resting firing rate (when distinct) is linked to the frequency at which it will exhibit peak sensitivity to an electrical vestibular stimulus.

\section{Nonlinear responses in monkey vestibular afferents and human neck MUs}

Although most vestibular afferents responded to the highfrequency electrical stimulation through modulation of their firing rate (Fig. $4 A$ ), examination of individual irregular afferent firing rate histograms revealed their firing tended to align (or lock) to specific phases of sinusoidal stimulus, particularly for frequencies $>\sim 100 \mathrm{~Hz}$ (see, insets in Fig. $4 A$, irregular afferent firing rate histograms). To quantify the extent of this phaselocking, we calculated the PLI (see Materials and Methods) in response to sinusoidal electrical stimuli up to $300 \mathrm{~Hz}$. As shown in Figure 6A, the average PLI for regular and irregular afferents increased as a function of frequency (main effect: $p<0.001$, $\left.F_{(12,224.8)}=52.1\right)$ and reached a plateau of $\sim 0.14$ and $\sim 0.18$ at a stimulus frequency of $\sim 150$ and $\sim 100 \mathrm{~Hz}$, respectively. On average, irregular afferents demonstrated higher PLIs than regular afferents at all frequencies but the difference in phase-locking between the two afferent types was not significant $(p=0.158$, $\left.F_{(1,19.9)}=2.2\right)$. More notably, the frequency at which PLIs plateaued for both afferent types matched the approximate frequency where sensitivity also plateaued and phase shifted from a lead to a lag response (Fig. 5A). This suggests that these changes in sensitivity and phase at high frequencies are linked to the increased tendency for afferents to phase-lock to the input stimulus.
Based on these results, we then examined whether we could observe any evidence of this vestibular afferent phase-locking in the downstream vestibularly-driven responses of neck motoneurons. We found that phase-locking in sternocleidomastoid MUs (Fig. 6B) increased with stimulus frequency only up to $75 \mathrm{~Hz}$ and then steadily decreased to a value of $\sim 0.050$ for the $300 \mathrm{~Hz}$ stimulus $\left(p<0.001, F_{(7,48.0)}=9.703\right)$. Similar responses were also observed in splenius capitis MUs in two subjects (Fig. 6B, inset). These results contrast with our above findings for vestibular afferents, which showed increasing phase-locking up to frequencies of $\sim 100-150 \mathrm{~Hz}$. Further, it is noteworthy that the decreasing tendency for neck MUs to phase lock at frequencies $>75 \mathrm{~Hz}$ aligned with the low-pass cutoff at $\sim 70-80 \mathrm{~Hz}$ observed in their response sensitivity (Fig. 3). Finally, the average PLI observed in sternocleidomastoid MUs across all equivalent frequencies (25$300 \mathrm{~Hz}$ ) was only $\sim 41-52 \%$ of the average PLI observed for vestibular afferents (Student's $t$ tests: $p<0.002$, Bonferroni's correction for multiple comparisons; $0.061 \pm 0.004$ vs $0.154 \pm$ 0.007 and $0.116 \pm 0.007$, for irregular and regular afferents, respectively). Overall, our results demonstrate that vestibular afferents show increasing sensitivity and phase lead with frequency in response to electrical stimulation up to the resting firing rate of the afferent $(100-150 \mathrm{~Hz})$, but thereafter sensitivity plateaus and afferent firing tends to phase-lock with the input stimulus. Further, we found that there is limited transmission of this high-frequency phase-locking observed in vestibular afferents to neck MUs.

\section{Human and monkey exhibit similar vestibulocollic reflex dynamics}

Thus far, we have shown that human vestibulocollic reflexes can be evoked by high-frequency vestibular afferent activity, but undergo low-pass neural filtering that diminishes their influence at frequencies above $75 \mathrm{~Hz}$. To validate our approach combining human neck muscle and nonhuman primate vestibular afferent responses, we next recorded multi-unit neck muscle activity in three monkeys during sinusoidal electrical stimuli. We found qualitatively similar results in the sensitivity and phase responses in monkeys relative to our human data (Fig. 7, top). Specifically, sensitivity increased with frequency to a peak before decreasing at 


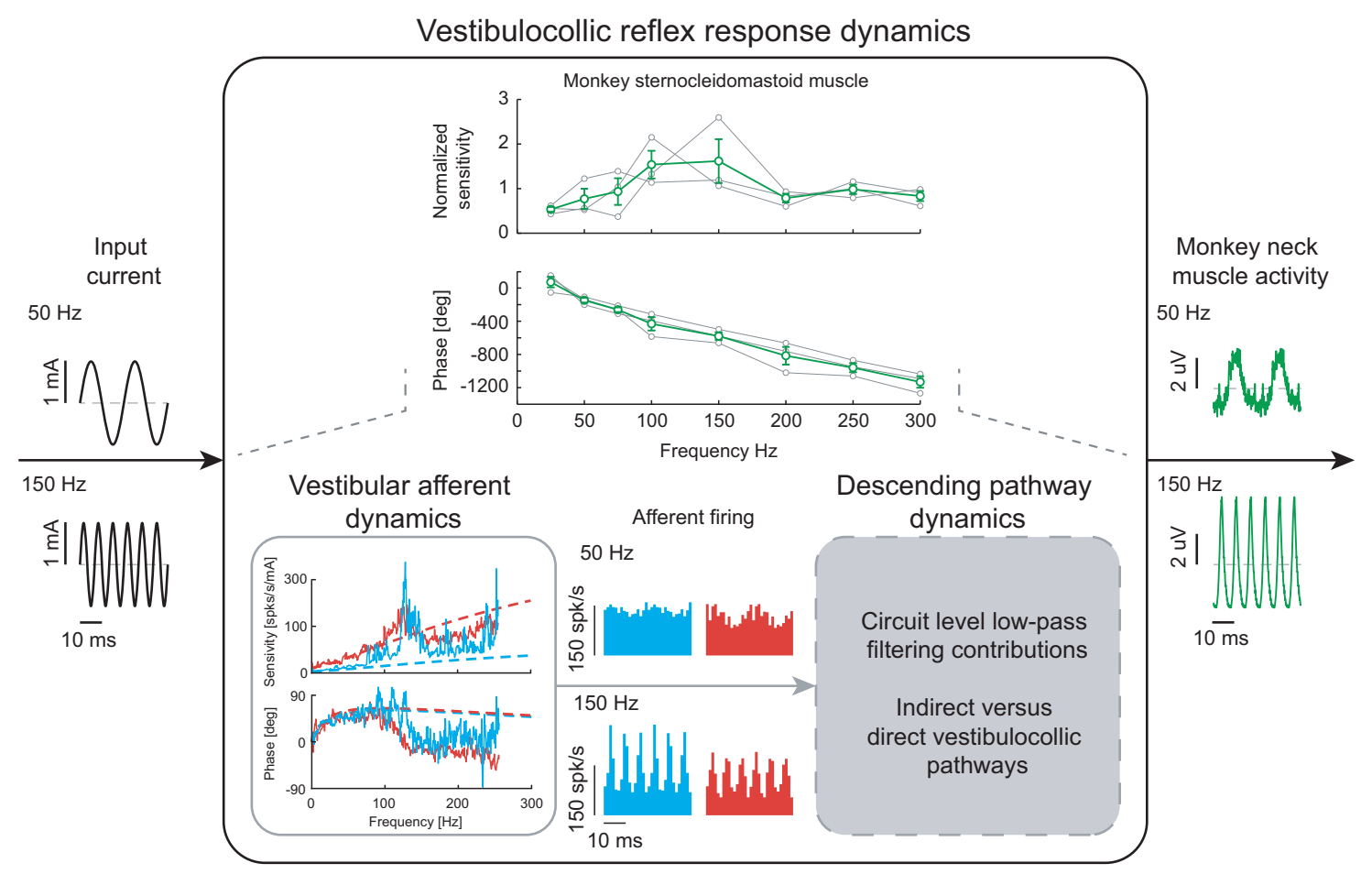

Figure 7. Schematic depicting the neural low-pass filtering observed in the monkey vestibulocollic reflex and the potential neural substrates responsible. Sensitivities from each monkey (gray lines in the top plot, $n=3$ ) were normalized to the mean sensitivity across all frequencies. Error bars indicate 1 SEM. The proposed filtering mechanisms limit both the increasing sensitivity of vestibular afferents at high frequencies and the tendency for vestibular afferents (regular: blue; irregular: red) to phase-lock at frequencies above the resting firing rate. Vestibular afferent dynamics during the stochastic stimulus are plotted together with transfer functions (dashed lines) obtained from Kwan et al. (2019).

higher frequencies, whereas phase decreased monotonically with frequency. On average, the sensitivity of vestibulocollic reflex responses recorded in the monkey sternocleidomastoid muscle peaked at $\sim 100-150 \mathrm{~Hz}$, corresponding to frequencies that were higher than the peak we observed for this same muscle in humans $(\sim 70-80 \mathrm{~Hz})$. Furthermore, response phases were comparable across species. These results suggest that the afferent and neural pathway dynamics subserving the VCR in humans and monkeys are similar, but that monkey VCRs can function across a higher frequency bandwidth compared with humans.

\section{Discussion}

The primary aim of this study was to gain new insight into the neural mechanisms that stabilize the head via the vestibulocollic reflex at high frequencies generated by dynamic events such as head impacts. We first found that human neck motoneurons responded to stimulation up to $300 \mathrm{~Hz}$, well above the highest frequencies reported to produce vestibular-evoked neck muscle activity $(75 \mathrm{~Hz})$ (Forbes et al., 2013). Sensitivities peaked at a cutoff of $\sim 70-80 \mathrm{~Hz}$ and then decreased at higher frequencies, while phase primarily decreased (i.e., lagged) across the entire bandwidth. Human single neck MU responses matched neck multi-unit EMG responses, confirming that the latter provides a robust estimate of vestibular-evoked neck muscle activity (Forbes et al., 2014, 2018). We next recorded from primary vestibular afferents in monkeys during comparable stimulation and found that they also responded to stimulation up to $300 \mathrm{~Hz}$. In particular, afferent sensitivities and phases increased (i.e., led) with frequencies up to values approaching their resting firing rates $(\sim 100 \mathrm{spk} / \mathrm{s})$. At higher frequencies, afferent sensitivities plateaued and responses tended to phase-lock with the input stimulus. Notably, this feature of afferent responses was not observed in human neck motoneurons, which instead showed significantly less phase-locking that also decreased (rather than increased) at frequencies $>75 \mathrm{~Hz}$. Finally, we recorded monkey neck muscle activity during vestibular stimulation and revealed qualitatively similar vestibulocollic reflex dynamics to those observed in humans. Taken together, our results indicate that the vestibular system is capable of transducing and transmitting highfrequency information that subsequently evokes neck muscle responses via vestibulocollic reflexes.

In both decerebrate and alert behaving animals, vestibular activity generated by imposed head motion (or electrical stimulation) evokes neck muscle responses that drive corrective head movements in the opposite direction (Ezure and Sasaki, 1978; Bilotto et al., 1982; Peterson et al., 1985; Goldberg and Peterson, 1986; Guitton et al., 1986; Dutia and Price, 1987; Wei and Angelaki, 2004; Forbes et al., 2014). Originating from both canal and otolith end organs (Sugiuchi et al., 2004; Uchino et al., 2005; Shinoda et al., 2006; Uchino and Kushiro, 2011), these VCR responses contribute to stabilization of the head in space. Notably, control systems analysis of the head-neck system lead to the suggestion that the VCR helps dampen the underdamped mechanics of the head-neck over a narrow band near the resonant frequency (1-3 Hz), while vision and head inertia make more significant contributions to head stabilization at lower and higher frequencies, respectively (Peng et al., 1996, 1997, 1999). Accordingly, the majority of studies investigating the VCR have been limited to frequencies up to $\sim 4 \mathrm{~Hz}$. Here, we further our understanding of the VCR by using an electrical stimuli to characterize vestibular afferent responses in monkeys as well as neck muscle responses in monkeys and humans up to frequencies reported during transient head-impacts ( $\sim 300 \mathrm{~Hz}$; Wu et al., 2016). Specifically, the 
increasing sensitivity of neck muscles to vestibular stimuli up to $\sim 70-80 \mathrm{~Hz}$ in humans and $\sim 100-150 \mathrm{~Hz}$ in monkeys demonstrates that peak VCR contributions to neck muscle activity occurs at frequencies likely produced by head transients (and not the resonant frequency of the head). Further, our current results suggest similarities in human and monkey neck muscle responses that help establish a nonhuman primate model for investigating VCR reflex pathways. This correspondence aligns with parallel observations in the statistics of natural vestibular stimuli experienced by both species (Carriot et al., 2017), as well as neck muscle recruitment profiles during eye-head gaze shifts (Corneil et al., 2001; Goonetilleke et al., 2015) and the morphology and function of many neck muscles (Kamibayashi and Richmond, 1998; Richmond et al., 2001).

As noted above, before this study the VCR has only been probed over a limited frequency range, and thus the response dynamics of the vestibulocollic pathway were not known (Fig. 7). Here, we found that primary vestibular afferents respond to vestibular stimuli up to $300 \mathrm{~Hz}$, whereas neck MUs comparatively demonstrate low-pass neural filtering of this input. This leads to the question: where does this filtering occur? In addition to direct projection from the vestibular nuclei, several indirect neural pathways transmit vestibular afferent signals (via the vestibular nuclei) to neck motoneurons, including the reticular formation and the interstitial nucleus of Cajal (Fig. 1) (for review, see Wilson and Schor, 1999; Goldberg and Cullen, 2011). Vestibular nuclei neurons, however, are an unlikely source of the observed neuronal filtering since similar to irregular vestibular afferents they tend to discriminate and encode self-motion stimuli through the precise timing of spikes (i.e., phase-locking) (Jamali et al., 2016; Jamali et al., 2019). An alternative possibility is that the indirect rather than direct vestibulospinal pathways are the main contributor to limiting the bandwidth of evoked neck motor responses since cutoffs of descending vestibular signals vary substantially across muscles (e.g., neck vs lower-limb muscles) (Forbes et al., 2013, 2015). Indeed, previous studies have emphasized that indirect pathways transmitting vestibular signals to the neck motoneurons make a dominant contribution to vestibulocollic reflexes (Shinoda and Yoshida, 1974; Wilson and Maeda, 1974; Fukushima et al., 1977; Ezure et al., 1978; Peterson et al., 1980; Bilotto et al., 1982; Fukushima et al., 1994). Further studies including multiple recordings along the different vestibulocollic pathways, will be required to parse these separate effects on the evoked neck muscle responses.

During most daily activities, the frequency content of head movements is limited to the physiologically relevant range of 0-30 Hz (Grossman et al., 1988; Huterer and Cullen, 2002; Carriot et al., 2014). The biomechanics and neural control of the body filter externally-induced environmental motion, causing the spectral power of head movement to rapidly decrease at frequencies above $\sim 5-10 \mathrm{~Hz}$ (Carriot et al., 2014). This occurs despite interactions with our environment, such as heel strikes while walking, that can produce vibrations in the foot with frequency content as high as $75 \mathrm{~Hz}$ (Simon et al., 1981). Direct head impacts and riding a roller coaster, however, can evoke head kinematics at frequencies above $100 \mathrm{~Hz}$ (Kuo et al., 2017; Wu et al., 2017). Massot et al. (2011) postulated that the increasing sensitivity of vestibular primary afferents with frequency, as also seen here up to their resting firing rate $(\sim 100-150 \mathrm{~Hz})$, compensates for the decreasing spectral power of vestibular input, thus broadening the capacity for vestibular afferents to encode highfrequency head movements. Indeed, both vestibular afferent and nuclei neurons encode natural self-motion through whitening over a 0 to $20 \mathrm{~Hz}$ bandwidth (i.e., maintaining constant spectral power across frequencies), a mechanism dependent on the increasing neural sensitivity and variability with frequency (Mitchell et al., 2018). The increased afferent and neck muscle sensitivity to the input stimulus from 0 to $100 \mathrm{~Hz}$ suggests that the mechanism underlying whitening in vestibular pathways could extend beyond the physiological range of head movement $(0-30 \mathrm{~Hz})$.

Our results have additional implications for the encoding strategies used by the different vestibular afferents as well as the neck MUs in response to the electrical stimulus. First, by stimulating vestibular afferents with a stochastic signal, we found a large peak in sensitivity for each afferent that corresponded to its resting firing rate, with the exception of highly irregular afferents $\left(\mathrm{CV}^{\star}>0.3\right)$ for which sensitivity progressively increased to a plateau. We speculate that an afferent's tendency to fire at a specific rate makes it inherently sensitive to input stimuli at that frequency. This may also explain why irregular afferents are more sensitive across all frequencies compared with regular afferents (e.g., Goldberg et al., 1984; Kwan et al., 2019). The tendency for irregular afferents to fire across a wide frequency bandwidth when at rest (Sadeghi et al., 2007; Massot et al., 2011) could make them more responsive to a wide range of frequencies compared with regular afferents. Second, the low-pass filtering behavior in the vestibulocollic pathways at frequencies above $\sim 75 \mathrm{~Hz}$ in humans (and $\sim 100-150 \mathrm{~Hz}$ in monkeys) may explain why phaselocking in neck motoneurons is relatively diminished compared with the plateau observed in the afferents at frequencies $>100-$ $150 \mathrm{~Hz}$. Although recent evidence indicates that temporal coding may be important for motor control (Sober et al., 2018), our findings suggest a minimal role for neck motoneuron phaselocking relative to rate coding in generating vestibulocollic reflexes over the large frequency range tested. Similar responses are expected across additional neck muscles given that highfrequency VCR responses are observed in deep and superficial neck muscles (Forbes et al., 2018).

Our present findings provide a new perspective on the contributions of vestibulocollic pathways to head stabilization in response to high-frequency transient head movements. Given the widespread influence of vestibular signals over all neck muscles (Forbes et al., 2018), which extends well beyond the highest frequencies of natural head movements $(\sim 30 \mathrm{~Hz}$; Grossman et al., 1988; Carriot et al., 2014), we propose that the transmission of high-frequency vestibular activity may serve to respond to transient events, such as those experienced during extreme sports or head impacts (Wu et al., 2017).

\section{References}

Bilotto G, Goldberg J, Peterson BW, Wilson VJ (1982) Dynamic properties of vestibular reflexes in the decerebrate cat. Exp Brain Res 47:343-352.

Boonstra TW, Breakspear M (2012) Neural mechanisms of intermuscular coherence: implications for the rectification of surface electromyography. J Neurophysiol 107:796-807.

Carriot J, Jamali M, Chacron MJ, Cullen KE (2014) Statistics of the vestibular input experienced during natural self-motion: implications for neural processing. J Neurosci 34:8347-8357.

Carriot J, Jamali M, Chacron MJ, Cullen KE (2017) The statistics of the vestibular input experienced during natural self-motion differ between rodents and primates. J Physiol 595:2751-2766.

Chen A, Khosravi-Hashemi N, Kuo C, Kramer JK, Blouin JS (2019) Development of a conversion model between mechanical and electrical vestibular stimuli. J Neurophysiol 123:548-559.

Corneil BD, Olivier E, Richmond FJ, Loeb GE, Munoz DP (2001) Neck muscles in the rhesus monkey. II. Electromyographic patterns of activation underlying postures and movements. J Neurophysiol 86:1729-1749.

Cullen KE (2019) Vestibular processing during natural self-motion: implications for perception and action. Nat Rev Neurosci 20:346-363. 
Cullen KE, Minor LB (2002) Semicircular canal afferents similarly encode active and passive head-on-body rotations: Implications for the role of vestibular efference. J Neurosci 22:RC226.

Dakin CJ, Son GM, Inglis JT, Blouin JS (2007) Frequency response of human vestibular reflexes characterized by stochastic stimuli. J Physiol 583:1117-1127.

Dakin CJ, Dalton BH, Luu BL, Blouin JS (2014) Rectification is required to extract oscillatory envelope modulation from surface electromyographic signals. J Neurophysiol 112:1685-1691.

Dale A, Cullen KE (2013) The nucleus prepositus predominantly outputs eye movement-related information during passive and active self-motion. J Neurophysiol 109:1900-1911.

Dutia MB, Price RF (1987) Interaction between the vestibulo-collic reflex and the cervico-collic stretch reflex in the decerebrate cat. J Physiol 387:19-30.

Ezure K, Sasaki S (1978) Frequency-response analysis of vestibular-induced neck reflex in cat .I. characteristics of neurla transmission from horizontal semicircular canal to neck motoneurons. J Neurophysiol 41:445-458.

Ezure K, Sasaki S, Uchino Y, Wilson VJ (1978) Frequency-response analysis of vestibular-induced neck reflex in cat II. functional significance of cervical afferents and polysynaptic descending pathways. J Neurophysiol 41:459-471.

Farina D, Negro F, Jiang N (2013) Identification of common synaptic inputs to motor neurons from the rectified electromyogram. J Physiol 591:24032418.

Fitzpatrick RC, Day BL (2004) Probing the human vestibular system with galvanic stimulation. J Appl Physiol 96:2301-2316.

Forbes PA, Dakin CJ, Vardy AN, Happee R, Siegmund GP, Schouten AC, Blouin JS (2013) Frequency response of vestibular reflexes in neck, back and lower limb muscles. J Neurophysiol 110:1869-1881.

Forbes PA, Siegmund GP, Happee R, Schouten AC, Blouin JS (2014) Vestibulocollic reflexes in the absence of head postural control. J Neurophysiol 112:1692-1702.

Forbes PA, Siegmund GP, Schouten AC, Blouin JS (2015) Task, muscle and frequency dependent vestibular control of posture. Front Integr Neurosci 8:94.

Forbes PA, Fice JB, Siegmund GP, Blouin JS (2018) Electrical vestibular stimuli evoke robust muscle activity in deep and superficial neck muscles in humans. Front Neurol 9:535.

Fukushima K, Ohashi T, Fukushima J (1994) Effects of chemical deactivation of the interstitial nucleus of cajal on the vertical vestibulo-collic reflex induced by pitch rotation in alert cats. Neurosci Res 20:281-286.

Fukushima Y, Igusa Y, Yoshida K (1977) Characteristics of responses of medial brain stem neurons to horizontal head angular acceleration and electrical stimulation of the labyrinth in the cat. Brain Res 120:564-570.

Goldberg J, Peterson BW (1986) Reflex and mechanical contributions to head stabilization in alert cats. J Neurophysiol 56:857-875.

Goldberg JM, Cullen KE (2011) Vestibular control of the head: possible functions of the vestibulocollic reflex. Exp Brain Res 210:331-345.

Goldberg JM, Smith CE, Fernández C (1984) Relation between discharge regularity and responses to externally applied galvanic currents in vestibular nerve afferents of the squirrel-monkey. J Neurophysiol 51:12361256.

Goonetilleke SC, Katz L, Wood DK, Gu C, Huk AC, Corneil BD (2015) Cross-species comparison of anticipatory and stimulus-driven neck muscle activity well before saccadic gaze shifts in humans and nonhuman primates. J Neurophysiol 114:902-913.

Grossman GE, Leigh RJ, Abel LA, Lanska DJ, Thurston SE (1988) Frequency and velocity of rotational head perturbations during locomotion. Exp Brain Res 70:470-476.

Guitton D, Kearney RE, Wereley N, Peterson BW (1986) Visual, vestibular and voluntary contributions to human head stabilization. Exp Brain Res 64:59-69.

Halliday DM, Farmer SF (2010) on the need for rectification of surface EMG. J Neurophysiol 103:35473547; author reply 3548-359.

Halliday DM, Rosenberg JR, Amjad AM, Breeze P, Conway BA, Farmer SF (1995) A framework for the analysis of mixed time series/point process data - theory and application to the study of physiological tremor, single motor unit discharges and electromyograms. Prog Biophys Mol Biol 64: 237-278.

Hayes AV, Richmond BJ, Optican LM (1982) A UNIX-based multiple process system for real-time data acquisition and control. In: Western Electronic
Show and Conference Proceedings, Vol 2, pp 1-10. Institute of Electrical and Electronics Engineers.

Huterer M, Cullen KE (2002) Vestibuloocular reflex dynamics during highfrequency and high-acceleration rotations of the head on body in rhesus monkey. J Neurophysiol 88:13-28.

Jamali M, Carriot J, Chacron MJ, Cullen KE (2013) Strong correlations between sensitivity and variability give rise to constant discrimination thresholds across the otolith afferent population. J Neurosci 33:1130211313.

Jamali M, Chacron MJ, Cullen KE (2016) Self-motion evokes precise spike timing in the primate vestibular system. Nat Commun 7:13229.

Jamali M, Carriot J, Chacron MJ, Cullen KE (2019) Coding strategies in the otolith system differ for translational head motion vs static orientation relative to gravity. eLife 8:e45573.

Jarvis MR, Mitra PP (2001) Sampling properties of the spectrum and coherency of sequences of action potentials. Neural Comput 13:717-749.

Johnston JA, Formicone G, Hamm TM, Santello M (2010) Assessment of across-muscle coherence using multi-unit vs. single-unit recordings. Exp Brain Res 207:269-282.

Kajikawa Y, Hackett TA (2005) Entropy analysis of neuronal spike train synchrony. J Neurosci Materials and Methods 149:90-93.

Kamibayashi LK, Richmond FJ (1998) Morphometry of human neck muscles. Spine 23:1314-1323.

Khosravi-Hashemi N, Forbes PA, Dakin CJ, Blouin JS (2019) Virtual signals of head rotation induce gravity-dependent inferences of linear acceleration. J Physiol 597:5231-5246.

Kuo C, Wu LC, Ye PP, Laksari K, Camarillo DB, Kuhl E (2017) Pilot findings of brain displacements and deformations during roller coaster rides. J Neurotrauma 34:3198-3205.

Kwan A, Forbes PA, Mitchell DE, Blouin JS, Cullen KE (2019) Neural substrates, dynamics and thresholds of galvanic vestibular stimulation in the behaving primate. Nat Commun 10:1904.

Lisberger SG, Pavelko TA (1986) Vestibular signals carried by pathways subserving plasticity of the vestibulo-ocular reflex in monkeys. J Neurosci 6:346-354

Luu BL, Muceli S, Saboisky JP, Farina D, Héroux ME, Bilston LE, Gandevia SC, Butler JE (2018) Motor unit territories in human genioglossus estimated with multichannel intramuscular electrodes. J Appl Physiol 124: 664-671.

Massot C, Chacron MJ, Cullen KE (2011) Information transmission and detection thresholds in the vestibular nuclei: single neurons vs. population encoding. J Neurophysiol 105:1798-1814.

McClelland VM, Cvetkovic Z, Mills KR (2012) Rectification of the EMG is an unnecessary and inappropriate step in the calculation of corticomuscular coherence. J Neurosci Materials and Methods 205:190-201.

Mitchell DE, Kwan A, Carriot J, Chacron MJ, Cullen KE (2018) Neuronal variability and tuning are balanced to optimize naturalistic self-motion coding in primate vestibular pathways. eLife 7:e43019.

Peng GC, Hain TC, Peterson BW (1996) A dynamical model for reflex activated head movements in the horizontal plane. Biol Cybern 75:309-319.

Peng GV, Hain TC and Peterson BW (1997) How is the head held up? Modeling mechanisms for head stability in the sagittal plane, In: Proceedings of 18th Annual International Conference of the IEEE Engineering in Medicine and Biology Society, pp 627-628, Amsterdam.

Peng GC, Hain TC, Peterson BW (1999) Predicting vestibular, proprioceptive, and biomechanical control strategies in normal and pathological head movements. IEEE Trans Biomed Eng 46:1269-1280.

Peterson BW, Fukushima K, Hirai N, Schor RH, Wilson VJ (1980) Responses of vestibulospinal and reticulospinal neurons to sinusoidal vestibular stimulation. J Neurophysiol 43:1236-1250.

Peterson BW, Goldberg J, Bilotto G, Fuller JH (1985) Cervicocollic reflex its dynamic properties and interaction with vestibular reflexes. J Neurophysiol 54:90-109.

Pintelon R, Schoukens J (2012) System identification: a frequency domain approach, Ed 2. New York: IEEE.

Richmond FJ, Singh K, Corneil BD (2001) Neck muscles in the rhesus monkey. I. Muscle morphometry and histochemistry. J Neurophysiol 86: $1717-1728$.

Rosenberg JR, Amjad AM, Breeze P, Brillinger DR, Halliday DM (1989) The Fourier approach to the identification of functional coupling between neuronal spike trains. Prog Biophys Mol Biol 53:1-31.

Sadeghi SG, Chacron MJ, Taylor MC, Cullen KE (2007) Neural variability, 
detection thresholds, and information transmission in the vestibular system. J Neurosci 27:771-781.

Schneider E, Glasauer S, Dieterich M (2002) Comparison of human ocular torsion patterns during natural and galvanic vestibular stimulation. J Neurophysiol 87:2064-2073.

Shinoda Y, Yoshida K (1974) Dynamic characteristics of responses to horizontal head angular acceleration in vestibuloocular pathway in cat. J Neurophysiol 37:653-673.

Shinoda Y, Sugiuchi Y, Izawa Y, Hata Y (2006) Long descending motor tract axons and their control of neck and axial muscles. Prog Brain Res 151:527-563.

Simon SR, Paul IL, Mansour J, Munro M, Abernethy PJ, Radin EL (1981) Peak dynamic force in human gait. J Biomech 14:817-822.

Sober SJ, Sponberg S, Nemenman I, Ting LH (2018) Millisecond spike timing codes for motor control. Trends Neurosci 41:644-648.

Straka H, Vibert N, Vidal PP, Moore LE, Dutia MB (2005) Intrinsic membrane properties of vertebrate vestibular neurons: function, development and plasticity. Prog Neurobiol 76:349-392.

Sugiuchi Y, Kakei S, Izawa Y, Shinoda Y (2004) Functional synergies among neck muscles revealed by branching patterns of single long descending motor-tract axons. Prog Brain Res 143:411-421.

Uchino Y, Kushiro K (2011) Differences between otolith- and semicircular canal-activated neural circuitry in the vestibular system. Neurosci Res 71:315-327.

Uchino Y, Sasaki M, Sato H, Bai R, Kawamoto E (2005) Otolith and canal integration on single vestibular neurons in cats. Exp Brain Res 164: 271-285.

Ward NJ, Farmer SF, Berthouze L, Halliday DM (2013) Rectification of EMG in low force contractions improves detection of motor unit coherence in the beta-frequency band. J Neurophysiol 110:1744-1750.

Watson SR, Colebatch JG (1998) Vestibulocollic reflexes evoked by shortduration galvanic stimulation in man. J Physiol 513:587-597.

Wei M, Angelaki DE (2004) Does head rotation contribute to gaze stability during passive translations? J Neurophysiol 91:1913-1918.

Wilkinson V, Malhotra A, Nicholas CL, Worsnop C, Jordan AS, Butler JE, Saboisky JP, Gandevia SC, White DP, Trinder J (2008) Discharge patterns of human genioglossus motor units during sleep onset. Sleep 31: 525-533.

Wilson VJ, Maeda M (1974) Connections between semicircular canals and neck motoneurons in cat. J Neurophysiol 37:346-357.

Wilson VJ, Schor RH (1999) The neural substrate of the vestibulocollic reflex: what needs to be learned. Exp Brain Res 129:483-493.

Wu LC, Laksari K, Kuo C, Luck JF, Kleiven S, 'Dale' Bass CR, Camarillo DB (2016) Bandwidth and sample rate requirements for wearable head impact sensors. J Biomech 49:2918-2924

Wu LC, Kuo C, Loza J, Kurt M, Laksari K, Yanez LZ, Senif D, Anderson SC, Miller LE, Urban JE, Stitzel JD, Camarillo DB (2017) Detection of american football head impacts using biomechanical features and support vector machine classification. Sci Rep 8:855. 